\title{
Macro-economic factors in credit risk calculations: including time-varying covariates in mixture cure models
}

\author{
Lore Dirick $^{1,2}$, Tony Bellotti ${ }^{3}$, Gerda Claeskens ${ }^{1,2}$, Bart Baesens ${ }^{2,4,5}$ \\ ${ }^{1}$ ORSTAT, Faculty of Economics and Business, KU Leuven, Belgium \\ ${ }^{2}$ Leuven Statistics Research Center (LSTAT), KU Leuven, Belgium \\ ${ }^{3}$ Department of Mathematics, Imperial College, London \\ ${ }^{4}$ LIRIS, Faculty of Economics and Business, KU Leuven, Belgium \\ ${ }^{5}$ School of Management, University of Southampton, UK \\ Lore.Dirick@kuleuven.be; A.Bellotti@imperial.ac.uk; \\ Gerda.Claeskens@kuleuven.be; Bart.Baesens@kuleuven.be
}

November 2, 2016

\begin{abstract}
The prediction of the time of default in a credit risk setting via survival analysis needs to take a high censoring rate into account. This rate is due to the fact that default does not occur for the majority of debtors. Mixture cure models allow the part of the loan population that is unsusceptible to default to be modelled, distinct from time of default for the susceptible population. In this paper, we extend the mixture cure model to include time-varying covariates. We illustrate the method via simulations and by incorporating macro-economic factors as predictors for an actual bank data set.
\end{abstract}

Keywords: Credit risk modeling; mixture cure model; time-varying covariates; macroeconomic factors; survival analysis. 


\section{Introduction}

With recent compliance guidelines such as the Basel accords, increased attention is devoted to more accurate calculations of the minimum amount of capital banks need to hold to provide a buffer against unexpected losses (Van Gestel and Baesens, 2008). Typically the probability of default (PD) of a certain loan applicant is estimated using classification techniques such as logistic regression. However, alternative methods have gained more importance in the recent credit scoring literature. In particular, survival analysis is an interesting tool as this method enables modeling of time until default, and not just whether a certain customer will default and the can be estimated over any time horizon.

Originally mainly used in medical science (see Collett, 2003; Cox and Oakes, 1984), survival analysis was first introduced in the credit scoring context by Narain (1992). While initially using fully parametric accelerated failure time survival models, other authors extended the idea of Narain (1992), using a Cox proportional hazards (PH) model (see Banasik et al., 1999), extensions on Cox PH models (Stepanova and Thomas, 2002) and including macro-economic variables (MVs) through time-varying covariates (TVCs) in Cox PH models (see Bellotti and Crook, 2009). Crook and Bellotti (2010) review several models for consumer loan credit risk modeling. Divino and Rocha (2013) compare survival analysis to the use of logistic regression models. In these papers, it is shown that survival analysis is a competitive method to logistic regression, and extending the Cox PH model further improves the accuracy of the estimated PD.

The survival function is $S(t)=P(T>t)$, which is the probability of observing an event time $T$ larger than some given $t$. A basic property of the survival function is that $S(t)=1-F(t)$, where $F(t)$ is the cumulative distribution function. Because of this relationship, $S(t)$ is assumed to go to zero as time proceeds, which means that all subjects under observation are expected to experience the event of interest eventually. As opposed to medical science where the event of interest is usually death, this property does not seem valid in the credit risk context, as a substantial part of the population will never experience default. In fact, it can be argued that unsusceptibility to default is the main reason behind the high censoring rate. The proportion of observations where default is not observed might in practice even exceed 95\%. Figure 1 clearly demonstrates this phenomenon for the credit 


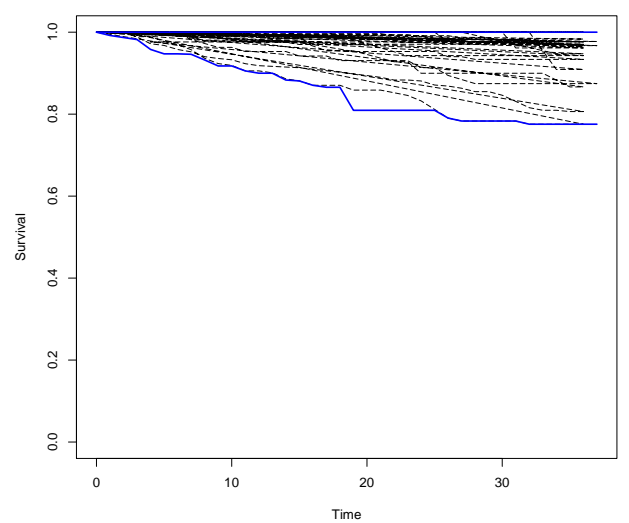

Figure 1: A sample of 20 estimated Kaplan-Meier estimates of the survival probability for the credit loan data as used in Section 6. The bounds indicate the region of all such estimated curves for this data example.

loan data set used in this paper (see Section 6). In this figure the continuous variables have been discretized by using their median, resulting in 128 estimated survival curves using the Kaplan-Meier estimator. To improve visibility, a random sample of 20 curves is shown in Figure 1, together with the bounds that the region of all 128 curves span. Clearly, the assumption that the survival function goes to zero does not hold. This implies that standard survival analysis methods and models do not apply well to this data set.

A remedy for this, the "mixture cure model", was initially proposed by Berkson and Gage (1952) and Farewell (1982) to model long-term survivors in the medical context. This model contains a logistic regression component, modeling "unsusceptibility" to the event of interest, and a survival component, modeling the survival times of an individual conditioning on susceptibility. While using parametric survival distributions in the survival component initially, Kuk and Chen (1992) extended the mixture cure model using nonparametric survival distributions (see also Peng and Dear, 2000; Sy and Taylor, 2000). Cai et al. (2012b) introduced the smcure-package in R (R Core Team, 2013) to estimate semi-parametric mixture cure models. This latter version of the mixture cure model was introduced in the credit risk context by Tong et al. (2012). Dirick et al. (2015) developed a model selection criterion for these models, and applied this to credit risk data.

While the use of TVCs has been investigated in (non-mixture) survival models, both in medical research (see among others Andersen, 1992) and in the credit context (see Bellotti 
and Crook, 2009), to our knowledge TVCs have not been implemented before in mixture cure models. In the present paper, we examine TVCs in these models, more specifically macro-economic factors, along with the usual time-independent covariates. The inclusion of TVC allows us to get a better understanding of why customers default. These insights can then be successfully adopted for ongoing credit risk monitoring (also called behavioural scoring in the industry) which help to determine provisions and capital buffers for both expected as well as unexpected losses.

The remainder of this paper is organized as follows. In Section 2, we give a short overview of different types of TVCs. In Sections 3 and 4, we discuss the mixture cure model with TVCs and the likelihood function, while computational details are placed in Appendix A. The simulation setup and results are discussed in Section 5, and a credit risk data example is presented in Section 6. Section 7 concludes.

\section{Time-varying covariates}

\subsection{Internal versus external TVCs}

TVCs can be segmented into two classes: internal and external TVCs; see, among others, Kalbfleisch and Prentice (2002, Chapter 6), Hosmer et al. (2008, Chapter 7) and Cortese and Andersen (2010). An internal TVC is one whose value is typically subject-specific and requires the subject to be under direct observation. An example of an internal TVC in the credit risk context is a customer's current account balance, or a patient's cholesterol level in the medical context. From the biomedical point of view, an internal covariate generally requires the survival of the individual for its existence. In this sense, the internal TVC-path carries direct information on the timing of the event if this event is death.

An external TVC does not require subjects to be under direct observation, nor does its existence depend on the occurrence of the event of interest. Examples of external TVCs are the inflation rate (in the credit risk context) and air pollution (in the biomedical context). In general, these TVCs are usually environmental factors that apply to all subjects under observation; however, subject-specific properties such as age are considered to be external as, given a subject's birth date, age can be determined at any time. A time-fixed covariate 
can be seen as a special case of an external time-dependent covariate, where its value is measured in advance and fixed for the entire study (e.g. the applicant's bureau score).

Formally, in a non-mixture survival context, denote $\boldsymbol{x}_{i}(t)=\left(x_{i 1}(t), \ldots, x_{i l}(t)\right)$ as the covariate vector at time $t$ for individuals $i=1, \ldots, n$. Additionally, denote the covariate history up to time $t$ : $X_{i}(t)=\left\{\boldsymbol{x}_{i}(u) ; 0 \leq u<t\right\}$. The available information for each observation $i$ is given by the time $T_{i}=\min \left(U_{i}, C_{i}\right)$, where $U_{i}$ denotes the true event time and $C_{i}$ is the censoring time, a corresponding censoring indicator $\delta_{i}=I\left(U_{i} \leq C_{i}\right)$ and $X_{i}\left(t_{i}\right)$, the covariate history until $t_{i}$.

A TVC is external when for all $v, t$, such that $0<v \leq t$ it satisfies the condition (Kalbfleisch and Prentice, 2002, Chapter 6)

$$
P(T \in[v, v+d v) \mid X(v), T \geq v)=P(T \in[v, v+d v) \mid X(t), T \geq v) .
$$

The rationale behind this condition is that although a time-dependent covariate may influence the event rate over time, its future path until any time $t$ is not affected by the occurrence of the event of interest at time $v$, or rather, in the interval $[v, v+d v)$ where $d v$ is a very small increment in time. The difference between internal and external covariates has great implications on survival function estimation. In presence of external covariates, the standard relationship between the survival function and the hazard function,

$$
S(t \mid X(t))=P(T>t \mid X(t))=\exp \left(-\int_{0}^{t} \lambda\{v \mid X(v)\} d v\right)
$$

holds, where $\lambda\{v \mid X(v)\}$ is the hazard function using the history $X(v)$. All TVCs described in this paper are macro-economic external variables, hence (2) can be used.

However, bear in mind that in case of internal covariates, extra attention should be given to the estimation of the survival function, see Andersen (1992) for a probabilistic model for survival function estimation in presence of internal TVCs. Because of the nature of internal TVCs and non-compliance to (1), however, estimation of instantaneous hazards is possible, but cumulative hazards and survival probabilities are no longer feasible through (2). To see this, we reconsider the example of the internal covariate cholesterol level in a study where the event of interest is death. From (2), any measurable cholesterol level value would indicate that the subject under investigation is still alive, hence, $S(t \mid X(t))=$ $P(T>t \mid X(t))=1$ given that $X(t)$ is measurable. For more information on this issue, we refer to Kalbfleisch and Prentice (2002, Chapter 6) and Fisher and Lin (1999). 


\subsection{Macro-economic factors}

Being a function of a (continuous) time $t$, TVCs can theoretically change continuously. This is approximately the case for some macro-economic variables (e.g. stock prices), others tend to be documented over longer periods of times such as unemployment rates (weekly, monthly or yearly). To manage TVCs in survival models, the observation period of each subject is split in several time-periods, which are defined by adjacent event times (Fox, 2002). Let $x_{i p}(t)$ (where $p \in\{1, \ldots, l\}$ ) be one specific time-dependent covariate, and let $B_{1}<\ldots<B_{m}$ be all the unique event or censoring times observed in the data set. To manage the data, subject $i$ must have exactly one TVC value for each of the intervals $\left\{\left(0, B_{1}\right],\left(B_{1}, B_{2}\right], \ldots,\left(B_{k_{i}-1}, B_{k_{i}}\right]\right\}$, where $k_{i} \in\{1, \ldots, m\}$ and $B_{k_{i}}=t_{i}$, hence each subject has its own set of TVC values until its own censoring or event time $t_{i}$.

Applied to default events in loans, these intervals represent the respective number of months a subject has been repaying until default or censoring. As a result, the TVCs are the monthly averages of specific macro-economic factors. This is denoted by replacing $x_{i p}(t)$ by $\bar{x}_{i p}(t)=\left(\bar{x}_{i p}\left(\left(0, B_{1}\right]\right), \bar{x}_{i p}\left(\left(B_{1}, B_{2}\right]\right), \ldots, \bar{x}_{i p}\left(\left(B_{k_{i}-1}, B_{k_{i}}\right]\right)\right)$, where $\bar{x}_{i p}\left(\left(B_{j-1}, B_{j}\right]\right)$ is the average value of TVC $p$ for subject $i$ over the time interval $\left(B_{j-1}, B_{j}\right]$.

\section{A mixture cure model with TVCs}

In a mixture cure model, cases are categorized into two groups: a group that will experience the event, and a group of so-called 'unsusceptible' cases that will not experience the event of interest. These groups are modeled using a mixture distribution where a logistic regression model provides a mixing proportion of the unsusceptible cases and where a survival model describes the cases susceptible to the event of interest (Tong et al., 2012). In the credit risk context, where the event of interest is loan default, every event-type that is not default (e.g. loan maturity, early repayment) is considered as censored. By consequence, there is heavy right-censoring and a large group of unsusceptible cases is expected to be present.

For each subject $i$, the censoring indicator $\delta_{i}$ denotes whether subject $i$ experiences the event of interest during the observation period $\left(\delta_{i}=1\right)$, or not $\left(\delta_{i}=0\right)$. This censoring indicator provides partial information on susceptibility; however, when an observation is 
censored, it is unclear whether the event will still occur after the observation period has terminated. Introducing a susceptibility indicator $Y_{i}$, where $Y_{i}=1$ when an observation is susceptible and $Y_{i}=0$ if not, three different combinations of $Y_{i}$ and $\delta_{i}$ are possible: (1) $Y_{i}=1$ and $\delta_{i}=1$ : uncensored and susceptible, the event takes place during the observation period; (2) $Y_{i}=1$ and $\delta_{i}=0$ : censored and susceptible, the event will take place, however is not observed; (3) $Y_{i}=0$ and $\delta_{i}=0$ : censored and unsusceptible, the event is not observed and will never take place. For each observation $i, T_{i}$ and $\delta_{i}$ are fully observed, $Y_{i}$ is only observed and equal to 1 when $\delta_{i}=1$.

\subsection{The model}

In a model with both time-dependent covariates $\boldsymbol{x}(t)$ and time-fixed covariates $\boldsymbol{z}$, the unconditional survival function of the mixture cure model is given by

$$
S(t \mid \boldsymbol{z}, \boldsymbol{x}(t))=\pi(\boldsymbol{z}) S(t \mid Y=1, \boldsymbol{z}, \boldsymbol{x}(t))+1-\pi(\boldsymbol{z}) .
$$

The 'incidence model', $\pi(\boldsymbol{z})=P(Y=1 \mid \boldsymbol{z})$, is the proportion of susceptible accounts given covariate vector $\boldsymbol{z}=\left(z_{1}, \ldots, z_{s}\right)^{\prime}$, modeled using a binary logit, with $\boldsymbol{b}=\left(b_{1}, \ldots, b_{s}\right)^{\prime}$

$$
\pi(\boldsymbol{z})=\exp \left(\boldsymbol{b}^{\prime} \boldsymbol{z}\right) /\left\{1+\exp \left(\boldsymbol{b}^{\prime} \boldsymbol{z}\right)\right\}
$$

Note that, in this part of the mixture cure model, only time-fixed covariates are incorporated. The conditional survival function is modeled using a semi-parametric proportional hazard regression model such that, with $\boldsymbol{\beta}=\left(\beta_{1}, \ldots, \beta_{s}\right)^{\prime}$,

$$
S(t \mid Y=1, \boldsymbol{z}, \boldsymbol{x}(t))=\exp \left(-\exp \left(\boldsymbol{\beta}^{\prime} \boldsymbol{z}+\boldsymbol{\beta}_{T}^{\prime} \boldsymbol{x}(t)\right) \int_{0}^{t} h_{0}(u \mid Y=1) d u\right),
$$

with $h_{0}$ the unspecified baseline hazard function, $\boldsymbol{x}(t)=\left(x_{1}(t), \ldots, x_{l}(t)\right)$ a $l$-vector of time-dependent covariates and $\boldsymbol{z}=\left(z_{1}, \ldots, z_{s}\right)$ a time-fixed covariate vector identical to the one in the incidence model. Note that from a theoretical point of view, the incidence and latency time-fixed covariate vectors may contain different variables; however in this paper, focusing on time-dependent covariates, these covariates are kept equal in all practical examples. For mixture cure models with different time-fixed covariate elements in latency and incidence models, we refer to Dirick et al. (2015). 


\subsection{The likelihood function}

To construct the likelihood function, specific attention should be devoted to the TVCs. Data management is the biggest challenge. We require that each time period (bounded by $B_{1}<\ldots<B_{m}$, see section 2.2) for a specific individual appears in a separate row in the data set (Fox, 2002). Denote $\lambda_{i, j}$ the interval-specific censoring indicator for interval $j \in\left\{1, \ldots, k_{i}\right\}$ of observation $i$. The complete likelihood, given full information on $Y$, is $L_{c}\left(\boldsymbol{b}, \boldsymbol{\beta}, \boldsymbol{\beta}_{t}\right)=\prod_{i=1}^{n}\left(1-\pi\left(\boldsymbol{z}_{i}\right)\right)^{\left(1-Y_{i}\right)} \pi\left(\boldsymbol{z}_{i}\right)^{Y_{i}} \prod_{j=1}^{k_{i}} h\left(t_{j} \mid Y_{i}=1, \boldsymbol{z}_{i}, \boldsymbol{x}_{i}\left(t_{j}\right)\right)^{\lambda_{i, j} Y_{i}} S\left(t_{j} \mid Y_{i}=1, \boldsymbol{z}_{i}, \boldsymbol{x}_{i}\left(t_{j}\right)\right)^{Y_{i}}$

where $h\left(t_{j} \mid \cdot\right)$ and $S\left(t_{j} \mid \cdot\right)$ are, respectively, the hazard and survival contributions at the time point given by the upper bound $B_{j}$ of the corresponding interval, and $\boldsymbol{x}_{i}\left(t_{j}\right)$ is the value of the TVC of observation $i$ in the interval $\left(B_{j-1}, B_{j}\right]$. The log likelihood function can be written as the sum of the latency and incidence log likelihoods,

$$
\log L_{c}\left(\boldsymbol{b}, \boldsymbol{\beta}, \boldsymbol{\beta}_{T} \mid \boldsymbol{z}, \boldsymbol{x}(t), Y\right)=\log L_{i n c}(\boldsymbol{b} \mid \boldsymbol{z}, Y)+\log L_{l a t}\left(\boldsymbol{\beta}, \boldsymbol{\beta}_{T} \mid \boldsymbol{z}, \boldsymbol{x}(t), Y\right),
$$

where

$$
\begin{array}{r}
\log L_{i n c}(\boldsymbol{b} \mid \boldsymbol{z}, Y)=\sum_{i=1}^{n}\left(1-Y_{i}\right)\left(1-\pi\left(\boldsymbol{z}_{i}\right)\right)+Y_{i} \pi\left(\boldsymbol{z}_{i}\right) \\
\log L_{l a t}\left(\boldsymbol{\beta}, \boldsymbol{\beta}_{T} \mid \boldsymbol{z}, \boldsymbol{x}(t), Y\right)=\sum_{i=1}^{n} \sum_{j=1}^{k_{i}} Y_{i} \lambda_{i, j} \log h\left(t_{j} \mid Y_{i}=1, \boldsymbol{z}_{i}, \boldsymbol{x}_{i}\left(t_{j}\right)\right) \\
+Y_{i} \log S\left(t_{j} \mid Y_{i}=1, \boldsymbol{z}_{i}, \boldsymbol{x}_{i}\left(t_{j}\right)\right) .
\end{array}
$$

As noted at the start of Section $3, Y_{i}$ is missing for the censored cases. As we do not have an exact expression for $\log L_{c}\left(\boldsymbol{b}, \boldsymbol{\beta}, \boldsymbol{\beta}_{T} \mid \boldsymbol{z}, \boldsymbol{x}(t), Y\right)$, the expectation maximization (EM) algorithm is used. This is an iterative procedure to find the maximum likelihood estimates using data that are incomplete (Dempster et al., 1977). We provide the needed adjustments to the algorithm to incorporate TVCs in mixture cure models, see the Appendix. 


\begin{tabular}{ccccc}
\hline & $\delta_{i}$ & $t_{i}$ & $\boldsymbol{z}_{1}$ & $\boldsymbol{z}_{2}$ \\
\hline obs 1 & 1 & 1 & -1 & 2 \\
obs 2 & 0 & 2 & 0.3 & 3 \\
obs 3 & 1 & 3 & 0.4 & 2.3 \\
\hline
\end{tabular}

\begin{tabular}{cccccccccc}
\hline & $B_{j-1}$ & $B_{j}$ & $\lambda_{i, j}$ & $\delta_{i}$ & $t_{i}$ & $\boldsymbol{z}_{1}$ & $\boldsymbol{z}_{2}$ & $\boldsymbol{x}_{1}(t)$ & $\boldsymbol{x}_{2}(t)$ \\
\hline obs 1 & 0 & 1 & 1 & 1 & 1 & -1 & 2 & 0.3 & -0.7 \\
obs 2 & 0 & 1 & 0 & 0 & 2 & 0.3 & 3 & 0.2 & 0.4 \\
obs 2 & 1 & 2 & 0 & 0 & 2 & 0.3 & 3 & 0.7 & -0.1 \\
obs 3 & 0 & 1 & 0 & 1 & 3 & 0.4 & 2.3 & 0.5 & -1 \\
obs 3 & 1 & 2 & 0 & 1 & 3 & 0.4 & 2.3 & 0.2 & -0.3 \\
obs 3 & 2 & 3 & 1 & 1 & 3 & 0.4 & 2.3 & 0.4 & 0.2 \\
\hline
\end{tabular}

Table 1: Example of the incidence versus latency model data structure. At the left: data structure for the binomial logit part of the mixture cure model, where no TVCs are present. At the right: the long data structure incorporating TVCs in the survival part of the model.

\section{Computational scheme}

\subsection{Data structure}

Including TVCs in the survival part of the mixture cure model requires rearrangement of the data. To make TVCs computationally feasible in a Cox PH model, each time period $\left(B_{j-1}, B_{j}\right]$ with $j=1, \ldots, k_{i}$ for each individual $i$ is represented as a single row in the data set (Fox, 2002). Note that the number of rows for each observation depends on the observation itself as $B_{k_{i}}=t_{i}$. The advantage of this data structure is that one can use the coxph-function in package survival in R (Therneau, 2014), using preamble "Surv(start, stop, default)" instead of the more familiar "Surv(time, default)".

The mixing proportions of the mixture cure model modeled by the binomial logit do not include TVCs, and using several lines per observation in this model part would lead to wrong estimates of $\boldsymbol{b}$. As a result, for the mixture cure model with TVCs, different data set structures are used depending on whether the respective calculations are performed on the latency or the incidence part of the model. An example of the 'short' (incidence) data structure versus the 'long' (latency) data structure is given in Table 1. To transform the short form of survival data into the long structure, Fox and Carvalho (2012) introduced the "unfold" function in the R-package RcmdrPlugin.survival. 


\subsection{Procedure}

The procedure consists of three main steps: initialization, the E-step and the M-step.

\subsubsection{Initialization}

1) Initialize $w$ : Initialize $w_{i}^{(0)}$ by taking $w_{i}^{(0)}=\delta_{i}$. Each observation has one $w_{i}^{(0)}$.

2) Initialize $\boldsymbol{b}$ : Fit a binomial logit model to $w_{i}^{(0)}$ using the 'short' data set and covariate vector $\boldsymbol{z}$, in order to retrieve an initial estimate $\hat{\boldsymbol{b}}^{(0)}$.

3) Initialize $\boldsymbol{\beta}$ and $\boldsymbol{\beta}_{T}$ : Obtain $\hat{\boldsymbol{\beta}}^{(0)}$ and $\hat{\boldsymbol{\beta}}_{T}^{(0)}$ using the coxph-function for the long survival data including TVCs. Use $w_{i}$ 's as weights in the model, matching $w_{i}$ with each line that corresponds with observation $i$.

4) Initialize $S_{0}(t)$ : Compute $\hat{S}_{0}^{(0)}(t)$ using formula (14).

\subsubsection{Expectation step}

1) Compute $\pi_{i}^{(1)}\left(z_{i}\right)$ for each $i$, using Formula (4), and $\hat{\boldsymbol{b}}^{(0)}$.

2) Compute $w_{i}^{(1)}$ for each $i$, using Formula (13), and $\hat{\beta}^{(0)}$. Note that the survival estimates used here, $\hat{S}^{(0)}\left(t_{i} \mid Y_{i}=1, \boldsymbol{z}_{i}, \boldsymbol{x}_{i}\left(t_{i}\right)\right)=\hat{S}_{0}^{(0)}\left(t_{i}\right)^{\exp \left(\hat{\boldsymbol{\beta}}^{\prime(0)} \boldsymbol{z}_{i}+\hat{\boldsymbol{\beta}}_{t}^{\prime(0)} \boldsymbol{x}_{i}\left(t_{i}\right)\right)}$, correspond for each observation to the estimate at the time of the last observation, hence the linear predictor consists of the TVC-values at time $t_{i}$.

\subsubsection{Maximization step}

1) Update $\boldsymbol{b}$ : Obtain a new estimate $\hat{\boldsymbol{b}}^{(1)}$ using the $w_{i}^{(1)}$ of the E-step when fitting the binomial logit model.

2) Update $\boldsymbol{\beta}$ and $\boldsymbol{\beta}_{T}$ : Obtain $\hat{\boldsymbol{\beta}}_{T}^{(1)}$ and $\hat{\boldsymbol{\beta}}^{(1)}$ including the $w_{i}^{(1)} \mathrm{s}$ as weights.

3) Update $S$ : Obtain a new estimate $\hat{S}^{(0)}(t)$ using formula (14).

The E and M-step are repeated with all updated estimates, until parameter convergence. The algorithm stops when the sum of the squared differences between $\left(\hat{\boldsymbol{\beta}}_{T}^{(r+1)}, \hat{\boldsymbol{\beta}}_{T}^{(r+1)}, \hat{\boldsymbol{b}}^{(r+1)}\right)$ and $\left(\hat{\boldsymbol{\beta}}_{T}^{(r)}, \hat{\boldsymbol{\beta}}_{T}^{(r)}, \hat{\boldsymbol{b}}^{(r)}\right)$ is smaller than a pre-specified value. 


\section{Simulation study}

\subsection{Simulating survival times with time-dependent covariates}

We include both time-fixed covariates $\boldsymbol{z}$ (associated with $\boldsymbol{b}$ and $\boldsymbol{\beta}$ ) and TVCs $\boldsymbol{x}(t)$ (associated with $\boldsymbol{\beta}_{T}$ ). When simulating survival times using an exponential distribution with only time-invariant covariates, the survival times and the cumulative hazard function can be defined piecewise, with $u \sim U(0,1)$,

$$
T=-\frac{\log (u)}{\lambda \exp \left(\boldsymbol{\beta}^{\prime} \boldsymbol{z}\right)}, \quad H(-\log (u), z)=\lambda \exp \left(\boldsymbol{\beta}^{\prime} \boldsymbol{z}\right)(-\log (u)) .
$$

Austin (2012) describes a method for generating survival times in the presence of TVCs which are constrained to be dichotomous variables with a limited number of changes between 0 and 1. For our purpose, we generalized this setting in two ways. (1) The TVC can change value from one time period to another, where a time period is defined by two adjacent event or censoring times. (2) The TVC can take any value, and does not need to be dichotomous.

In the simulation we set the boundaries that define the TVC intervals as follows. We denote by $B_{j}$ the timepoints where the covariate values change. Note that $j \in\{1, \ldots, m\}$ with $m \leq n-1$, with $n$ the number of cases, as both the event and censoring times are unique in a simulation study when using continuous time distributions. As a notational convention, we use $x\left(t_{j}\right)$ for the value of the time-dependent covariate in the interval $\left(B_{j-1}, B_{j}\right]$. In a generalization of the simulation method by Austin (2012), the cumulative hazard function is given by

$$
\begin{aligned}
& H(v, z, x(t)) \\
& = \begin{cases}\lambda \exp \left(\boldsymbol{\beta}^{\prime} \boldsymbol{z}+\boldsymbol{\beta}_{T}^{\prime} \boldsymbol{x}\left(t_{1}\right)\right)(v) & \text { if } v \leq B_{1} \\
\lambda \exp \left(\boldsymbol{\beta}^{\prime} \boldsymbol{z}\right)\left[\exp \left(\boldsymbol{\beta}_{T}^{\prime} \boldsymbol{x}\left(t_{1}\right)\right) B_{1}+\exp \left(\boldsymbol{\beta}_{T}^{\prime} \boldsymbol{x}\left(t_{2}\right)\right)\left(v-B_{1}\right)\right] & \text { if } B_{1}<v \leq B_{2} \\
\vdots & \\
\lambda \exp \left(\boldsymbol{\beta}^{\prime} \boldsymbol{z}\right)\left[\sum_{j=1}^{m}\left(\exp \left(\boldsymbol{\beta}_{T}^{\prime} \boldsymbol{x}\left(t_{j}\right)\right)\left(B_{j}-B_{j-1}\right)\right)+\exp \left(\boldsymbol{\beta}_{T}^{\prime} \boldsymbol{x}\left(t_{m+1}\right)\right)\left(v-B_{m}\right)\right] & \text { if } B_{m}<v\end{cases}
\end{aligned}
$$

where $v=-\log (u)$. The domain of the cumulative hazard function can be divided into mutually exclusive intervals $D_{1}=\left(0, B_{1}\right], D_{2}=\left(B_{1}, B_{2}\right], \ldots, D_{m+1}=\left(B_{m}, \infty\right)$, with the 
corresponding ranges of the cumulative hazard functions,

$$
\begin{aligned}
R_{1} & =\left(0, \lambda \exp \left(\boldsymbol{\beta}^{\prime} \boldsymbol{z}+\boldsymbol{\beta}_{T}^{\prime} \boldsymbol{x}\left(t_{1}\right)\right) B_{1}\right] ; \\
R_{2} & =\left(\lambda \exp \left(\boldsymbol{\beta}^{\prime} \boldsymbol{z}+\boldsymbol{\beta}_{T}^{\prime} \boldsymbol{x}\left(t_{1}\right)\right) B_{1}, \lambda \exp \left(\boldsymbol{\beta}^{\prime} \boldsymbol{z}\right)\left\{\exp \left(\boldsymbol{\beta}_{T}^{\prime} \boldsymbol{x}\left(t_{1}\right)\right) B_{1}+\exp \left(\boldsymbol{\beta}_{T}^{\prime} \boldsymbol{x}\left(t_{2}\right)\right)\left(B_{2}-B_{1}\right)\right\}\right] ; \\
\vdots & \\
R_{m+1} & =\left(\lambda \exp \left(\boldsymbol{\beta}^{\prime} \boldsymbol{z}\right) \sum_{j=1}^{m}\left(\exp \left(\boldsymbol{\beta}_{T}^{\prime} \boldsymbol{x}\left(t_{j}\right)\right)\left(B_{j}-B_{j-1}\right)\right), \infty\right) .
\end{aligned}
$$

By inverting each of the piecewise components of the cumulative hazard function we can simulate the survival time as $H^{-1}(v, z, x)$ with

$$
\begin{aligned}
& H^{-1}(v, z, x(t)) \\
& = \begin{cases}\frac{v}{\lambda \exp \left(\boldsymbol{\beta}^{\prime} \boldsymbol{z}+\boldsymbol{\beta}_{T}^{\prime} \boldsymbol{x}\left(t_{1}\right)\right)} & \text { if } v \in R_{1} \\
\frac{v-\lambda \exp \left(\boldsymbol{\beta}^{\prime} \boldsymbol{z}+\boldsymbol{\beta}_{T}^{\prime} \boldsymbol{x}\left(t_{1}\right)\right) B_{1}+\lambda \exp \left(\boldsymbol{\beta}^{\prime} \boldsymbol{z}+\boldsymbol{\beta}_{T}^{\prime} \boldsymbol{x}\left(t_{2}\right)\right) B_{1}}{\lambda \exp \left(\boldsymbol{\beta}^{\prime} \boldsymbol{z}+\boldsymbol{\beta}_{T}^{\prime} \boldsymbol{x}\left(t_{2}\right)\right)} & \text { if } v \in R_{2} \\
\vdots & \text { if } v \in R_{m+1} . \\
\frac{v+\lambda \exp \left(\boldsymbol{\beta}^{\prime} \boldsymbol{z}\right)\left\{\sum_{j=1}^{m}\left(-\exp \left(\boldsymbol{\beta}_{T}^{\prime} \boldsymbol{x}\left(t_{j}\right)\right)\left(B_{j}-B_{j-1}\right)\right)+\exp \left(\boldsymbol{\beta}_{T}^{\prime} \boldsymbol{x}\left(t_{m+1}\right)\right)\left(B_{m}\right)\right\}}{\lambda \exp \left(\boldsymbol{\beta}^{\prime} \boldsymbol{z}+\boldsymbol{\beta}_{T}^{\prime} \boldsymbol{x}\left(t_{m+1}\right)\right)} & \end{cases}
\end{aligned}
$$

\subsection{Simulation setup and results}

\subsubsection{Uncorrelated time-varying covariates}

The probability of being unsusceptible is generated using a logistic model where $\pi(z)=$ $\exp \left(\boldsymbol{b}^{\prime} \boldsymbol{z}\right) /\left\{1+\exp \left(\boldsymbol{b}^{\prime} \boldsymbol{z}\right)\right\}$, and the survival times of the susceptible cases are generated using an exponential distribution with $\lambda=0.7$. We generate two uncorrelated time-fixed covariates $z_{1} \sim N(1.5,0.6)$ and $z_{2} \sim \operatorname{bin}(1,0.5)$, and two time-dependent covariates $x_{1}(t) \sim$ $N(2,0.5)$ and $x_{2}(t) \sim N(0.8,0.5)$.

Different simulation settings are implemented in order to explore different aspects of model behaviour. In settings I, II and III, $\boldsymbol{\beta}=(-1.2,1)^{\prime}$ and $\boldsymbol{\beta}_{T}=(1,-0.7)^{\prime}$, while in settings IV, V and VI, $\boldsymbol{\beta}=(1,-3)^{\prime}$ and $\boldsymbol{\beta}_{T}=(-0.5,0.9)^{\prime}$. Susceptibility is managed through the vector $\boldsymbol{b}$, which is $(2,0.5,-2.3)$ for settings I and IV (low censoring/susceptibility), $(-0.5,0.8,-1.5)$ for settings II and V (medium censoring/susceptibility) and $(-1.5,0.5,-2)$ for settings III and VI (high censoring/susceptibility). Censoring times are generated from an exponential distribution using $\lambda=0.1$ for low and medium settings, and $\lambda=0.2$ for the 
high censoring settings. For each of the six settings, we take $n=300, n=500$ and $n=1000$ and use 100 replications for each sample size. Note that the TVC can theoretically change value $n-1$ times. To imitate real-data situations, we constrained the TVCs to change values at most 60 times, as the data sets we typically use have a loan term of 60 months (i.e. five years) or less.

In Table 2 and 4, the true generating parameter values are shown, as well as the mean of the parameter estimates, the standard errors over the 100 simulation runs for each sample size and the absolute biases and the mean squared errors between the parameter estimates and the true values.

From Tables 2 and 4, we see that higher censorship leads to higher MSE, while a larger $n$ tends to lower the MSE. A small $n$ in combination with high censorship led to a degeneration of some of the $\hat{\beta}_{2}$ estimates for Setting IV. This issue does not translate to the bias, despite the fact that the same effect of sample size and censorship seems to apply. Even under large censorship, the parameter estimates related to the TVCs $\left(\hat{\beta}_{T 1}\right.$ and $\hat{\beta}_{T 2}$ ) are more stable according to the simulations. The abundant information in the TVCs (for each case in our simulation 40 to 60 different values for one TVC) enables an accurate parameter estimation.

Table 3 shows the results of a comparison with the standard Cox proportional hazard regression model. The results are for settings I-III, for sample size $n=1000$ and should be compared to the corresponding cases in Table 4. Other settings give a similar inferior performance as compared to the mixture cure models, although those results are not shown. In particular the time independent variables are estimated with a bias.

\subsubsection{Correlated time-varying covariates}

In real life, macro-economic factors are all linked and influence each other. To mimic this behaviour, setting VII takes TVCs that are highly correlated. Time-fixed covariates have the same distributions as for the previous settings, $n=1000$ and the generating parameters are as in setting I. However, this time we included three TVCs that are highly correlated, 


\begin{tabular}{|c|c|c|c|c|c|c|c|c|c|c|c|c|c|c|}
\hline & & & \multicolumn{4}{|c|}{$n=300$} & \multicolumn{4}{|c|}{$n=500$} & \multicolumn{4}{|c|}{$n=1000$} \\
\hline & & true & avg est & avg sd & bias & MSE & avg est & avg sd & bias & MSE & avg est & avg sd & bias & MSE \\
\hline \multirow{7}{*}{ 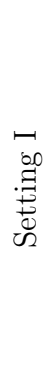 } & $\hat{b}_{0}$ & 2.00 & 2.151 & 0.474 & 0.151 & 0.384 & 2.151 & 0.368 & 0.151 & 0.249 & 2.076 & 0.254 & 0.076 & 0.094 \\
\hline & $\hat{b}_{1}$ & 0.5 & 0.481 & 0.269 & 0.019 & 0.105 & 0.532 & 0.213 & 0.032 & 0.077 & 0.540 & 0.153 & 0.040 & 0.027 \\
\hline & $\hat{b}_{2}$ & -2.3 & -2.349 & 0.290 & 0.049 & 0.219 & -2.439 & 0.225 & 0.139 & 0.235 & -2.368 & 0.148 & 0.068 & 0.079 \\
\hline & $\hat{\beta}_{1}$ & -1.2 & -1.137 & 0.125 & 0.063 & 0.032 & -1.152 & 0.101 & 0.048 & 0.018 & -1.150 & 0.070 & 0.050 & 0.009 \\
\hline & $\hat{\beta}_{2}$ & 1.0 & 0.898 & 0.132 & 0.102 & 0.043 & 0.876 & 0.108 & 0.124 & 0.033 & 0.894 & 0.077 & 0.106 & 0.022 \\
\hline & $\hat{\beta}_{T 1}$ & 1.0 & 0.983 & 0.132 & 0.017 & 0.016 & 0.990 & 0.102 & 0.010 & 0.010 & 0.988 & 0.072 & 0.012 & 0.005 \\
\hline & $\hat{\beta}_{T 2}$ & -0.7 & -0.687 & 0.132 & 0.013 & 0.023 & -0.692 & 0.101 & 0.008 & 0.014 & -0.691 & 0.071 & 0.009 & 0.006 \\
\hline \multirow{7}{*}{ 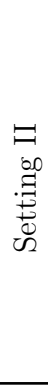 } & $\hat{b}_{0}$ & -0.50 & -0.372 & 0.332 & 0.128 & 0.203 & -0.453 & 0.255 & 0.047 & 0.120 & -0.421 & 0.183 & 0.079 & 0.088 \\
\hline & $\hat{b}_{1}$ & 0.8 & 0.796 & 0.165 & 0.004 & 0.086 & 0.889 & 0.120 & 0.089 & 0.063 & 0.896 & 0.109 & 0.096 & 0.050 \\
\hline & $\hat{b}_{2}$ & -1.5 & -1.571 & 0.231 & 0.071 & 0.093 & -1.612 & 0.181 & 0.112 & 0.094 & -1.655 & 0.123 & 0.155 & 0.081 \\
\hline & $\hat{\beta}_{1}$ & -1.2 & -1.072 & 0.160 & 0.128 & 0.064 & -1.088 & 0.132 & 0.112 & 0.040 & -1.029 & 0.084 & 0.171 & 0.042 \\
\hline & $\hat{\beta}_{2}$ & 1.0 & 0.911 & 0.185 & 0.089 & 0.094 & 0.896 & 0.142 & 0.104 & 0.045 & 0.838 & 0.097 & 0.162 & 0.049 \\
\hline & $\hat{\beta}_{T 1}$ & 1.0 & 0.991 & 0.170 & 0.009 & 0.029 & 0.976 & 0.130 & 0.024 & 0.019 & 1.006 & 0.091 & 0.006 & 0.006 \\
\hline & $\hat{\beta}_{T 2}$ & -0.7 & -0.680 & 0.168 & 0.020 & 0.045 & -0.673 & 0.129 & 0.027 & 0.024 & -0.715 & 0.090 & 0.015 & 0.009 \\
\hline \multirow{7}{*}{ 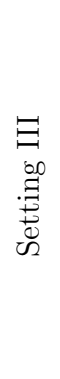 } & $\hat{b}_{0}$ & -1.50 & -1.316 & 0.396 & 0.184 & 0.421 & -1.391 & 0.315 & 0.109 & 0.247 & -1.379 & 0.213 & 0.121 & 0.126 \\
\hline & $\hat{b}_{1}$ & 0.5 & 0.449 & 0.227 & 0.051 & 0.147 & 0.494 & 0.145 & 0.006 & 0.114 & 0.521 & 0.093 & 0.021 & 0.061 \\
\hline & $\hat{b}_{2}$ & -2.0 & -2.020 & 0.338 & 0.020 & 0.226 & -2.048 & 0.265 & 0.048 & 0.163 & -2.091 & 0.187 & 0.091 & 0.072 \\
\hline & $\hat{\beta}_{1}$ & -1.2 & -1.000 & 0.293 & 0.200 & 0.241 & -1.080 & 0.233 & 0.120 & 0.134 & -1.064 & 0.148 & 0.136 & 0.069 \\
\hline & $\hat{\beta}_{2}$ & 1.0 & 0.921 & 0.387 & 0.079 & 0.284 & 0.848 & 0.320 & 0.152 & 0.250 & 0.866 & 0.204 & 0.134 & 0.096 \\
\hline & $\hat{\beta}_{T 1}$ & 1.0 & 0.974 & 0.311 & 0.026 & 0.102 & 0.978 & 0.230 & 0.022 & 0.064 & 0.943 & 0.159 & 0.057 & 0.025 \\
\hline & $\hat{\beta}_{T 2}$ & -0.7 & -0.697 & 0.306 & 0.003 & 0.100 & -0.693 & 0.231 & 0.007 & 0.076 & -0.686 & 0.159 & 0.014 & 0.027 \\
\hline
\end{tabular}

Table 2: Simulation study. True values, averaged estimates, standard deviation, bias and mean squared error for different settings and sample sizes. Setting I: unsusceptible= $22.63 \%$, censoring $=32.89 \%$; Setting II: unsusceptible $=50.9 \%$, censoring $=58.26 \%$; Setting III: unsusceptible $=80.70 \%$, censoring $=86.08 \%$.

$\boldsymbol{x}(t)=\left(x_{1}(t), x_{2}(t), x_{3}(t)\right)^{\prime}$ with mean, covariance matrix and correlation matrix

$$
\mu=\left(\begin{array}{c}
2 \\
0.8 \\
-0.7
\end{array}\right) ; \quad \Sigma=\left(\begin{array}{ccc}
0.7 & 0.8 & 0.8 \\
0.8 & 1.2 & 0.8 \\
0.8 & 0.8 & 1.0
\end{array}\right) ; \quad \rho=\left(\begin{array}{ccc}
1 & 0.873 & 0.956 \\
0.873 & 1 & 0.730 \\
0.956 & 0.730 & 1
\end{array}\right)
$$

In setting VII, censoring times are generated using an exponential distribution with $\lambda=$ 0.15. Although mean standard errors of the TVCs are larger as compared to the time-fixed covariates and the results in Tables 2 and 4 (the right panel for Setting I in particular), 


\begin{tabular}{rrrrrr}
\hline Setting I & true value & avg est & avg std & Bias & MSE \\
\hline$\hat{\beta}_{1}$ & -1.2 & -0.3315 & 0.0645 & 0.8685 & 0.7579 \\
$\hat{\beta}_{2}$ & 1.0 & -0.4704 & 0.0801 & 1.4704 & 2.1687 \\
$\hat{\beta}_{T 1}$ & 1.0 & 0.9216 & 0.0777 & 0.0784 & 0.0118 \\
$\hat{\beta}_{T 2}$ & -0.7 & -0.6530 & 0.0777 & 0.0470 & 0.0082 \\
\hline Setting II & true value & avg est & avg std & Bias & MSE \\
\hline$\hat{\beta}_{1}$ & -1.2 & 0.1224 & 0.0808 & 1.3224 & 1.7546 \\
$\hat{\beta}_{2}$ & 1.0 & -0.7599 & 0.1038 & 1.7599 & 3.1088 \\
$\hat{\beta}_{T 1}$ & 1.0 & 0.9022 & 0.0985 & 0.0978 & 0.0199 \\
$\hat{\beta}_{T 2}$ & -0.7 & -0.6492 & 0.0986 & 0.0508 & 0.0130 \\
\hline Setting III & true value & avg value & avg std & Bias & MSE \\
\hline$\hat{\beta}_{1}$ & -1.2 & 0.0274 & 0.1419 & 1.2274 & 1.5267 \\
$\hat{\beta}_{2}$ & 1.0 & -1.5891 & 0.2205 & 2.5891 & 6.7396 \\
$\hat{\beta}_{T 1}$ & 1.0 & 0.9003 & 0.1708 & 0.0997 & 0.0322 \\
$\hat{\beta}_{T 2}$ & -0.7 & -0.6490 & 0.1710 & 0.0510 & 0.0266 \\
\hline
\end{tabular}

Table 3: Simulation study with $n=1000$ and using the Cox proportional hazard regression model. True values, averaged estimates, standard deviation, bias and mean squared error for different settings.

Table 5 show that biases and mean squared errors are not notably larger for $b$ and $\beta_{T}$. Estimators of $\beta_{1}$ and $\beta_{2}$ seem to have a higher bias. This result is due to the larger gap between the percentage of censored and unsusceptible cases $(39.01-22.72 \%=16.29 \%)$. Throughout our simulations, it has become clear that the estimates of $\beta$ deteriorate when this gap becomes bigger than $10 \%$. The $\beta_{T}$ estimates, however, do not seem to be affected.

\section{Data set with macro-economic variables}

The data used was provided by a major Belgian financial institution. The sample, consisting of 20000 personal loans with a fixed loan term of 36 months, spanned a period of loans that were initiated between January 2004 and May 2014. Among these 20000 loans, 839 ended in 


\begin{tabular}{|c|c|c|c|c|c|c|c|c|c|c|c|c|c|c|}
\hline & & & \multicolumn{4}{|c|}{$n=300$} & \multicolumn{4}{|c|}{$n=500$} & \multicolumn{4}{|c|}{$n=1000$} \\
\hline & & true & avg est & avg sd & bias & MSE & avg est & avg sd & bias & MSE & avg est & avg sd & bias & MSE \\
\hline \multirow{7}{*}{ 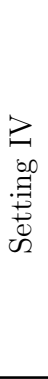 } & $\hat{b}_{0}$ & 2.00 & 2.232 & 0.489 & 0.232 & 0.617 & 2.120 & 0.355 & 0.120 & 0.328 & 2.121 & 0.244 & 0.121 & 0.146 \\
\hline & $\hat{b}_{1}$ & 0.50 & 0.499 & 0.229 & 0.001 & 0.199 & 0.527 & 0.174 & 0.027 & 0.112 & 0.475 & 0.120 & 0.025 & 0.056 \\
\hline & $\hat{b}_{2}$ & -2.30 & -2.429 & 0.423 & 0.129 & 0.372 & -2.367 & 0.324 & 0.067 & 0.193 & -2.195 & 0.217 & 0.105 & 0.122 \\
\hline & $\hat{\beta}_{1}$ & 1.00 & 1.012 & 0.131 & 0.012 & 0.024 & 0.978 & 0.098 & 0.022 & 0.017 & 0.990 & 0.068 & 0.010 & 0.006 \\
\hline & $\hat{\beta}_{2}$ & -3.00 & -2.983 & 0.255 & 0.017 & 0.126 & -2.960 & 0.172 & 0.040 & 0.052 & -3.016 & 0.118 & 0.016 & 0.023 \\
\hline & $\hat{\beta}_{T 1}$ & -0.50 & -0.441 & 0.135 & 0.059 & 0.030 & -0.388 & 0.103 & 0.112 & 0.021 & -0.406 & 0.072 & 0.094 & 0.014 \\
\hline & $\hat{\beta}_{T 2}$ & 0.90 & 0.907 & 0.137 & 0.007 & 0.027 & 0.895 & 0.105 & 0.005 & 0.013 & 0.895 & 0.073 & 0.005 & 0.006 \\
\hline \multirow{7}{*}{ 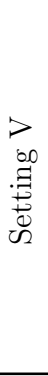 } & $\hat{b}_{0}$ & -0.50 & -0.464 & 0.305 & 0.036 & 0.216 & -0.419 & 0.233 & 0.081 & 0.134 & -0.470 & 0.166 & 0.030 & 0.066 \\
\hline & $\hat{b}_{1}$ & 0.80 & 0.809 & 0.186 & 0.009 & 0.089 & 0.799 & 0.141 & 0.001 & 0.047 & 0.797 & 0.098 & 0.003 & 0.023 \\
\hline & $\hat{b}_{2}$ & -1.50 & -1.455 & 0.249 & 0.045 & 0.123 & -1.496 & 0.187 & 0.004 & 0.097 & -1.415 & 0.132 & 0.085 & 0.049 \\
\hline & $\hat{\beta}_{1}$ & 1.00 & 1.007 & 0.164 & 0.007 & 0.035 & 0.970 & 0.124 & 0.030 & 0.026 & 0.983 & 0.087 & 0.017 & 0.012 \\
\hline & $\hat{\beta}_{2}$ & -3.00 & -2.910 & 0.319 & 0.090 & 0.135 & -2.925 & 0.227 & 0.075 & 0.115 & -2.985 & 0.141 & 0.015 & 0.045 \\
\hline & $\hat{\beta}_{T 1}$ & -0.50 & -0.424 & 0.167 & 0.076 & 0.042 & -0.406 & 0.128 & 0.094 & 0.024 & -0.395 & 0.090 & 0.105 & 0.022 \\
\hline & $\hat{\beta}_{T 2}$ & 0.90 & 0.852 & 0.169 & 0.048 & 0.040 & 0.886 & 0.130 & 0.014 & 0.019 & 0.882 & 0.091 & 0.018 & 0.010 \\
\hline \multirow{7}{*}{ 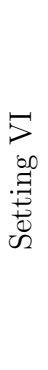 } & $\hat{b}_{0}$ & -1.50 & -1.351 & 0.380 & 0.149 & 0.324 & -1.465 & 0.292 & 0.035 & 0.214 & -1.415 & 0.202 & 0.085 & 0.118 \\
\hline & $\hat{b}_{1}$ & 0.50 & 0.459 & 0.226 & 0.041 & 0.104 & 0.496 & 0.176 & 0.004 & 0.064 & 0.480 & 0.121 & 0.020 & 0.035 \\
\hline & $\hat{b}_{2}$ & -2.00 & -2.226 & 0.396 & 0.226 & 0.567 & -2.315 & 0.313 & 0.315 & 0.372 & -2.190 & 0.207 & 0.190 & 0.176 \\
\hline & $\hat{\beta}_{1}$ & 1.00 & 1.094 & 0.261 & 0.094 & 0.131 & 0.962 & 0.196 & 0.038 & 0.070 & 0.977 & 0.150 & 0.023 & 0.024 \\
\hline & $\hat{\beta}_{2}$ & -3.00 & -3.219 & 125.338 & 0.219 & 9.256 & -3.114 & 94.559 & 0.114 & 9.057 & -2.576 & 0.335 & 0.424 & 0.503 \\
\hline & $\hat{\beta}_{T 1}$ & -0.50 & -0.416 & 0.273 & 0.084 & 0.085 & -0.404 & 0.210 & 0.096 & 0.070 & -0.415 & 0.145 & 0.085 & 0.033 \\
\hline & $\hat{\beta}_{T 2}$ & 0.90 & 0.917 & 0.278 & 0.017 & 0.088 & 0.843 & 0.213 & 0.057 & 0.049 & 0.888 & 0.145 & 0.012 & 0.023 \\
\hline
\end{tabular}

Table 4: Simulation study. True values, averaged estimates, standard deviation, bias and mean squared error for different settings and sample sizes. Setting IV: unsusceptible= $22.56 \%$, censoring $=36.51 \%$; Setting V: unsusceptible $=51.07 \%$, censoring $=58.14 \%$; Setting VI: unsusceptible $=80.67 \%$, censoring $=83.45 \%$.

a default and 5376 in an early repayment. As could be expected given the rather short term of the loans, the loan amounts are sized accordingly. The distribution of loan amounts is shown in Figure 2. In the sample, 76.28\% of the loan amounts are below 10000 and $97.85 \%$ below 20000 . In each of the models that are discussed, seven time-independent covariates described in Table 6 (further information about these covariates cannot be disclosed due to reasons of commercial confidentiality) are included as a baseline. As these variables are used in a Cox PH model, a graphical check, through parallel discretized Kaplan-Meier curves, 


\begin{tabular}{cccccc}
\hline & true & avg est & avg sd & bias & MSE \\
\hline$\hat{b}_{0}$ & 2 & 2.0834 & 0.2654 & 0.0834 & 0.1273 \\
$\hat{b}_{1}$ & 0.5 & 0.6053 & 0.1562 & 0.1053 & 0.0592 \\
$\hat{b}_{2}$ & -2.3 & -2.3036 & 0.1682 & 0.0036 & 0.1363 \\
$\hat{\beta}_{1}$ & -1.2 & -1.0529 & 0.0728 & 0.1471 & 0.0303 \\
$\hat{\beta}_{2}$ & 1 & 0.6866 & 0.0769 & 0.3134 & 0.1189 \\
$\hat{\beta}_{T 1}$ & 1 & 0.9276 & 0.2716 & 0.0724 & 0.1048 \\
$\hat{\beta}_{T 2}$ & -0.7 & -0.6663 & 0.0924 & 0.0337 & 0.0120 \\
$\hat{\beta}_{T 3}$ & 0.5 & 0.5036 & 0.1673 & 0.0036 & 0.0367 \\
\hline
\end{tabular}

Table 5: Results for simulation setting VII, true values, averaged estimates, standard deviation, bias and mean squared error. Unsusceptible $=22.72 \%$, censoring $=39.01 \%$.

\begin{tabular}{lll}
\hline & Description & Type \\
\hline$z_{1}$ & Annual income (per 1000) & continuous \\
$z_{2}$ & Age & continuous \\
$z_{3}$ & Monthly child allowance $(\mathrm{Y} / \mathrm{N})$ & categorical \\
$z_{4}$ & Number of years at current address & continuous \\
$z_{5}$ & Total employment years & continuous \\
$z_{6}$ & Bureau score & continuous \\
$z_{7}$ & Mortgage on real estate $(\mathrm{Y} / \mathrm{N})$ & categorical \\
\hline
\end{tabular}

Table 6: Credit loan data, time-independent covariates. Covariates $\boldsymbol{z}_{1}, \boldsymbol{z}_{2}, \boldsymbol{z}_{4}$ and $\boldsymbol{z}_{5}$ are mean-centered and $\boldsymbol{z}_{6} \log$ transformed.

for the proportional hazards assumption was performed and showed that this assumption was satisfied. Additionally, six macro-economic factors were gathered through the online database from the Belgian National Bank (NBB, 2015). A TVC-value was retained for each month in the years 2004 until 2014, correcting for both trend and seasonality by taking the yearly difference for each TVC (e.g. the TVC-value for unemployment in August 2008 is the difference between its value in August 2008 and August 2007). As some macro-economic 


\begin{tabular}{|c|c|c|c|}
\hline & Type & Lag & Description \\
\hline $\bar{x}_{1}(t)$ & Interest Rate & 6 months & $\begin{array}{l}\text { As the interest rates of the Belgian financial institution were not disclosed, } \\
\text { the minimum bid interest rate was chosen. This refers to the minimum } \\
\text { interest rate at which counterparties may place their bids for refinancing } \\
\text { operations. }\end{array}$ \\
\hline $\bar{x}_{2}(t)$ & BEL 20 index & none & $\begin{array}{l}\text { The benchmark stock market index of Euronext Brussels, consisting of ten } \\
\text { to twenty (depending on the period) companies that are traded at Brussels } \\
\text { Stock Exchange. The TVC is expressed as the difference between the index } \\
\text { of the current period and the previous year, divided by } 1000 \text {. }\end{array}$ \\
\hline $\bar{x}_{3}(t)$ & Consumer confidence & none & $\begin{array}{l}\text { Monthly survey on a variable sample of } 1850 \text { households conducted by the } \\
\text { National Bank. The survey, harmonized at European level, supplies infor- } \\
\text { mation on the appreciation of the consumers regarding the progress of the } \\
\text { economy in general and regarding their own situation in particular. }\end{array}$ \\
\hline $\bar{x}_{4}(t)$ & Gross Domestic product & none & $\begin{array}{l}\text { Growth in the Belgian Gross Domestic Product with respect to the same } \\
\text { period in the previous year (GDP growth is documented quarterly). }\end{array}$ \\
\hline $\bar{x}_{5}(t)$ & Inflation rate & 6 months & $\begin{array}{l}\text { Percentage changes in consumer price compared to the corresponding period } \\
\text { of the previous year. }\end{array}$ \\
\hline $\bar{x}_{6}(t)$ & Unemployment & 6 months & $\begin{array}{l}\text { Harmonised data derived from the Labour Force Survey (LFS, population } \\
\text { older than } 15 \text { years), monthly adjusted by using the administrative national } \\
\text { unemployment figures, in accordance with the Eurostat methodology. }\end{array}$ \\
\hline
\end{tabular}

Table 7: Time-dependent covariates $\bar{x}_{1}(t)$ to $\bar{x}_{6}(t)$ are differential macro-economic factors that change month by month. A specific TVC is the difference between the nominal macroeconomic factor value in a specific month and the same factor twelve months earlier.

factors may have a delayed effect on default, time lags of six months were introduced for the TVCs of market interest rate, inflation rate and unemployment. Hence, we examine the effect of the inflation rate in, say, February 2005 on possible default in August 2005.

As the financial crisis of 2007-2008 was fully covered in the sample, we examined the effect of the crisis on the number of defaults. Figure 3 shows the proportion of defaults in each month, represented by the number of defaults in each month divided by the total number of loans that were actually running in that month (and were hence "at risk"). Evidence of elevated defaults in the period 2007-01 to 2008-12 is present in the histogram, with an average monthly default proportion of 0.0021 in the latter period versus an overall average of 0.0017 . 


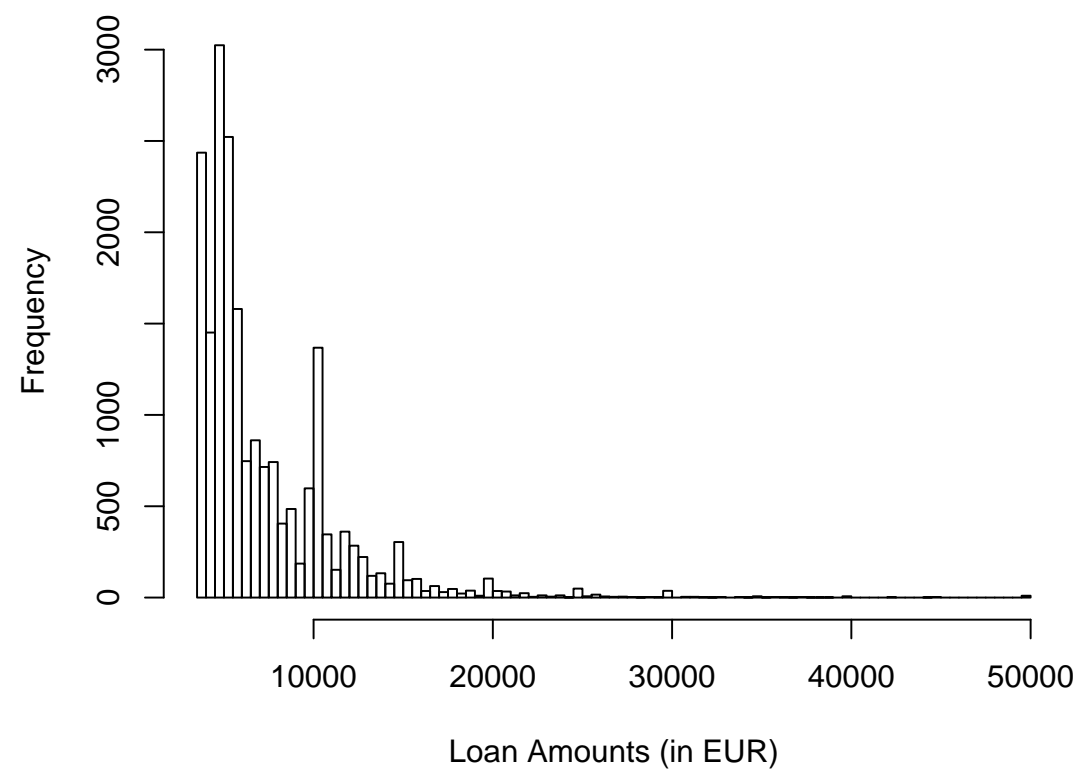

Figure 2: Data example. Histogram representing the distribution of the loan amounts. Only 3 loans had amounts over 50000 EUR (not shown in the plot for reasons of clarity).

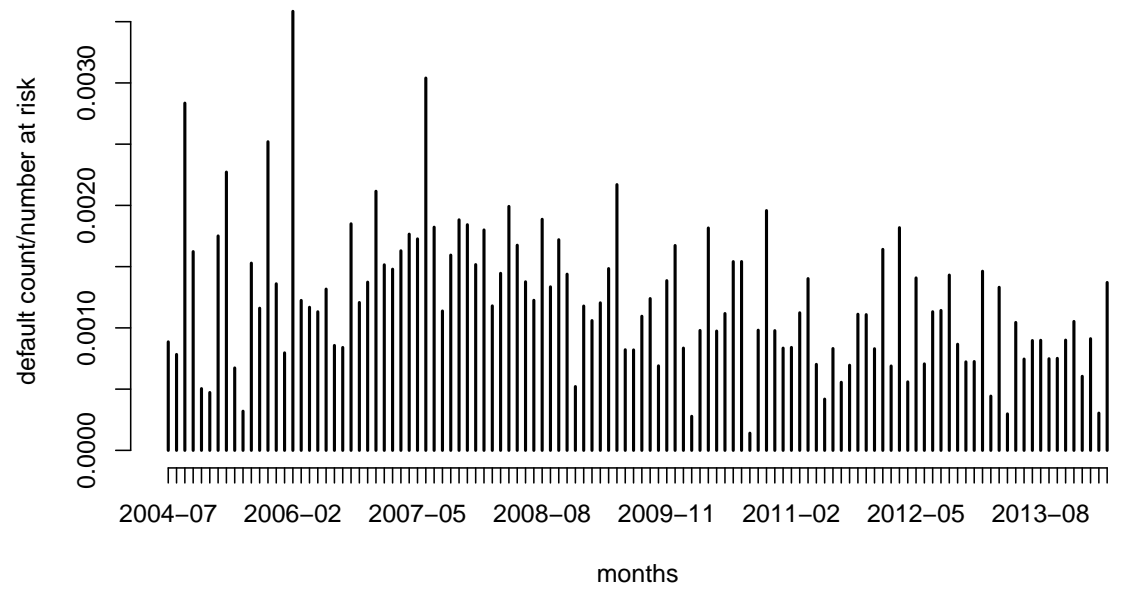

Figure 3: Data example. Proportion of defaults in each month as a fraction of the total number of loans that were actually running in each month.

\subsection{Data analysis using the mixture cure model}

Information about the time-independent and time-dependent covariates can be found in Tables 6 and 7 respectively. Several mixture cure models, each including the same seven 
time-independent covariates, and three or four different TVCs (leading to thirty-five models in total) were analyzed. For each of these models, a corrected version of the Akaike information criterion (named complete-data $\mathrm{AIC}$ or the $A I C_{c d}$ ) was computed. This $A I C_{c d}$ is based on the converged complete-data log likelihood $Q(\widehat{\Theta} \mid \widehat{\Theta})$ instead of the standard log likelihood and can be computed through

$$
A I C_{c d}=-2 Q(\widehat{\Theta} \mid \widehat{\Theta})+2 d+2 \operatorname{trace}\left\{D M\left(I_{d}-D M\right)^{-1}\right\}
$$

where $d$ is the length of the parameter vector, $I_{d}$ is a $d \times d$ identity matrix and $D M$ is the matrix rate of convergence of the EM algorithm, which is automatically computed when using the SEM-algorithm (Meng and Rubin, 1991). The $A I C_{c d}$ in the mixture cure context is discussed in detail in Dirick et al. (2015).

When performing model selection one typically fits a series of models that are deemed appropriate for the data at hand. A statistical model search via information criteria differs from fitting one full model and reducing this by considering individual significance tests, which leads to well-known multiple testing and pre-testing problems (Danilov and Magnus, 2004). The efficiency of AIC makes it preferable to other such criteria (e.g. the Schwarz Bayesian information criterion) when the model is to be used to make predictions (see for example Claeskens and Hjort, 2008, Chapter 2), which is the purpose of credit risk modeling. Statistical individual significance is not considered in this process.

A general result from the analysis of the thirty-five models was that both BEL 20 index and the interest rate tended to have a significant impact on default. The other macroeconomic factors, however, did not have a significant effect. The parameter information for the three best models with respect to the $A I C_{c d}$-values, along with the model that only contains the seven time-independent covariates, are given in Table 8. As a general result, the $A I C_{c d}$ clearly improves by including TVCs in the models. On the other hand, a lower $A I C_{c d}$ does not guarantee a model with significant TVCs, as can be seen in the $A I C_{c d}$ "best" model. For an explanation on how particular parameter estimates affect default, we look at the model with significant effects for the interest rate and BEL 20 index. Residential stability $\left(z_{4}\right)$, length of employment $\left(z_{5}\right)$, a higher bureau score $\left(z_{6}\right)$ and the presence of a mortgage $\left(z_{7}\right)$ lead to a lower susceptibility ( $\hat{\boldsymbol{b}}$ is negative). The corresponding negative estimates $\hat{\boldsymbol{\beta}}$ for these four variables indicate a longer time until default as well. Both $\hat{\boldsymbol{b}}$ and 


\begin{tabular}{|c|c|c|c|c|c|c|c|c|c|c|c|c|c|c|c|}
\hline$A I C_{c d}$ & & (int) & $z_{1}$ & $z_{2}$ & $z_{3}$ & $z_{4}$ & $z_{5}$ & $z_{6}$ & $z_{7}$ & IR & BEL 20 & cons conf & GDP & infl & unempl \\
\hline \multirow[t]{3}{*}{ no TVC } & $b$ & 2.492 & 0.016 & 0.005 & -0.076 & -0.023 & -0.039 & -1.083 & -1.319 & & & & & & \\
\hline & & $(0.015)$ & $(0.002)$ & $(0.001)$ & $(0.033)$ & $(0.001)$ & $(0.002)$ & $(0.038)$ & $(0.026)$ & & & & & & \\
\hline & & $* * *$ & $* * *$ & $* * *$ & $* *$ & $* * *$ & $* * *$ & $* * *$ & $* * *$ & & & & & & \\
\hline \multirow[t]{3}{*}{30126.98} & $\boldsymbol{\beta}$ & & -0.000 & -0.011 & -0.074 & -0.024 & -0.023 & -0.615 & -0.488 & & & & & & \\
\hline & & & $(0.005)$ & $(0.003)$ & $(0.077)$ & $(0.004)$ & $(0.006)$ & $(0.104)$ & $(0.092)$ & & & & & & \\
\hline & & & ns & $* * *$ & $\mathrm{~ns}$ & $* * *$ & $* * *$ & $* * *$ & $* * *$ & & & & & & \\
\hline \multirow[t]{3}{*}{ best } & $b$ & 3.55 & 0.046 & 0.058 & 0.192 & 0.009 & -0.07 & -1.406 & -2.153 & & & & & & \\
\hline & & $(0.085)$ & $(0.002)$ & $(0.002)$ & $(0.037)$ & $(0.002)$ & $(0.002)$ & $(0.04)$ & $(0.033)$ & & & & & & \\
\hline & & $* * *$ & $* * *$ & $* * *$ & $* * *$ & $* * *$ & $* * *$ & $* * *$ & $* * *$ & & & & & & \\
\hline \multirow[t]{3}{*}{26685.22} & $\boldsymbol{\beta}$ & & -0.011 & -0.026 & -0.188 & -0.036 & -0.016 & -0.519 & -0.119 & & & -0.003 & -0.011 & 0.001 & \\
\hline & & & $(0.005)$ & $(0.003)$ & $(0.076)$ & $(0.004)$ & $(0.006)$ & $(0.101)$ & $(0.077)$ & & & $(0.005)$ & $(0.021)$ & $(0.041)$ & \\
\hline & & & $*$ & $* * *$ & $*$ & $* * *$ & $* *$ & $* * *$ & $\mathrm{~ns}$ & & & $\mathrm{~ns}$ & ns & $\mathrm{ns}$ & \\
\hline \multirow[t]{3}{*}{ second best } & $b$ & 2.861 & 0.035 & 0.034 & 0.067 & -0.003 & -0.059 & -1.23 & -1.829 & & & & & & \\
\hline & & $(0.079)$ & $(0.002)$ & $(0.001)$ & $(0.036)$ & $(0.002)$ & $(0.002)$ & $(0.039)$ & $(0.032)$ & & & & & & \\
\hline & & $* * *$ & $* * *$ & $* * *$ & . & $*$ & $* * *$ & $* * *$ & $* * *$ & & & & & & \\
\hline \multirow[t]{3}{*}{27743.78} & $\beta$ & & -0.008 & -0.021 & -0.133 & -0.031 & -0.016 & -0.543 & -0.188 & 0.071 & -0.14 & & 0.009 & -0.033 & \\
\hline & & & $(0.005)$ & $(0.003)$ & $(0.077)$ & $(0.004)$ & $(0.005)$ & $(0.1)$ & $(0.075)$ & $(0.036)$ & $(0.057)$ & & $(0.022)$ & $(0.041)$ & \\
\hline & & & . & $* * *$ & . & $* * *$ & $* *$ & $* * *$ & $*$ & . & $*$ & & ns & $\mathrm{ns}$ & \\
\hline \multirow[t]{3}{*}{ third best } & $b$ & 3.309 & 0.028 & 0.023 & 0 & -0.011 & -0.048 & -1.22 & -1.588 & & & & & & \\
\hline & & $(0.072)$ & $(0.002)$ & $(0.001)$ & $(0.034)$ & $(0.002)$ & $(0.002)$ & $(0.037)$ & $(0.033)$ & & & & & & \\
\hline & & $* * *$ & $* * *$ & $* * *$ & ns & $* * *$ & $* * *$ & $* * *$ & $* * *$ & & & & & & \\
\hline \multirow[t]{3}{*}{29378.38} & $\beta$ & & -0.002 & -0.015 & -0.108 & -0.029 & -0.025 & -0.659 & -0.489 & & & -0.003 & & 0.005 & -0.051 \\
\hline & & & $(0.005)$ & $(0.002)$ & $(0.074)$ & $(0.004)$ & $(0.006)$ & $(0.102)$ & $(0.085)$ & & & $(0.005)$ & & $(0.031)$ & $(0.05)$ \\
\hline & & & ns & $* * *$ & ns & $* * *$ & $* * *$ & $* * *$ & $* * *$ & & & ns & & ns & ns \\
\hline
\end{tabular}

Table 8: Data example. Parameter estimates for the three best models according to their $A I C_{c d^{-}}$values and for the model without TVCs. $(\cdot)$ significant at the $10 \%$ level, $(*)$ at the $5 \%$ level, $(* *)$ at the $1 \%$ level and $(* * *)$ significant at the $0.1 \%$ level.

$\hat{\boldsymbol{\beta}}$ indicate that debtors with more job and residential stability, as well as a higher bureau score and having a real estate mortgage tend to be less prone to default. The effect of annual income $\left(z_{1}\right)$, age $\left(z_{2}\right)$ and presence of a monthly child allowance $\left(z_{3}\right)$ is less clear: with positive estimates $\hat{\boldsymbol{b}}$, susceptibility to default is increased, but the negative estimates $\hat{\boldsymbol{\beta}}$ indicate delayed default. Comparing parameter estimates over all models, it seems that the only variable that leads to significant parameter estimates that have consistently opposite signs for the two model parts, is variable $z_{2}$ (age). It is not surprising that the signs often are the same because the underlying intuition behind both model parts is risk of default. However, a higher risk of being susceptible to default does not necessarily mean that default will occur sooner. This is what is observed for the variable age: it seems that susceptibility to default increases slightly with age, but older people seem to go into default 
closer to the maturity of the loan. When looking at the TVCs, logically, a higher interest rate leads to an increase in default hazard $\left(\hat{\boldsymbol{\beta}}_{\boldsymbol{T}}\right.$ is positive), and a better state of the BEL 20 index leads to a decrease in default (negative sign). With insignificant effects of the gross domestic product and inflation rate on default, there is no conclusive effect of these TVCs on default. Baseline hazard estimates (not shown here) are consistently larger for the model without TVCs as compared to the estimates for the other models. The model that has been selected as the best gives the lowest baseline hazard estimates, compared to the whole range of models.

The magnitude of the macro-economic effects is small, which is an expected result, as the models are averaging across people that are more or less likely to be affected by the economy. Small effects at individual level will potentially aggregate to large effects at portfolio level, as macro-economic factors will have the same effect for all individuals. This result also follows previous studies such as Bellotti and Crook (2009).

\subsection{The effect of the covariate value on the survival probability}

To illustrate the effect of a certain covariate combination on the fitted probabilities of default, we looked at two different covariate combinations in the data set and fitted survival probabilities using the best model in Table 8. Additionally, a combination of two different time-dependent covariate vectors was examined. The resulting covariate values can be found in Table 9. In covariate combinations 1a and 2a, the lowest observed consumer confidence level was attached, along with the lowest observed GDP and the highest inflation rate. These values lead to the worst possible survival probabilities in presence of the timefixed covariates for covariate 1 or 2 (because of the negative sign for the former two TVCparameters, and the positive sign of the latter). For covariate combinations $2 \mathrm{a}$ and $2 \mathrm{~b}$, the best observed consumer confidence level was attached, along with the best observed GDP and the worst observed inflation rate.

The fitted survival probabilities for multiples of six months using the covariate values in Table 9 and the best model in Table 8 are shown in Table 10. Fitting the model using covariate combinations $1 \mathrm{a}$ and $2 \mathrm{a}$ leads to lower survival probabilities compared to $1 \mathrm{~b}$ and $2 \mathrm{~b}$ respectively, and the time-fixed covariates for covariate combinations $1 \mathrm{a}$ and $1 \mathrm{~b}$ lead to 
higher survival probabilities compared to $2 \mathrm{a}$ and $2 \mathrm{~b}$. The difference in TVCs can make up to nearly $3 \%$ difference in the 36 th month of the loan.

It is important to note that these fitted probabilities are purely intended as an illustration of the effect certain covariate values might have on the probability of default (ie 1 survival probability). In reality forecasting and stress testing can only be done using economic forecasts and not the actual values of the macro-economic factors. Secondly, TVCs change from one month to another, whereas for this illustration, TVCs were assumed to stay at their maximal (minimal) level for the entire loan duration.

\begin{tabular}{ccccccccccc}
\hline Example & $z_{1}$ & $z_{2}$ & $z_{3}$ & $z_{4}$ & $z_{5}$ & $z_{6}$ & $z_{7}$ & cons conf & GDP & infl \\
\hline 1a & 6.46 & 10.17 & 1 & -3.73 & 13.80 & 2.29 & 0 & -21 & -6.30 & 5.91 \\
1b & 6.46 & 10.17 & 1 & -3.73 & 13.80 & 2.29 & 0 & 20 & 7.20 & -1.69 \\
2a & -11.49 & 8.17 & 0 & -7.81 & -8.28 & 1.61 & 1 & -21 & -6.30 & 5.91 \\
2b & -11.49 & 8.17 & 0 & -7.81 & -8.28 & 1.61 & 1 & 20 & 7.20 & -1.69 \\
\hline
\end{tabular}

Table 9: Data example. Two random covariate combinations for the non-TVCs were selected from the data set. For each of them, the lowest (highest) observed TVC-level for consumer confidence and GDP, and the highest observed TVC-level for the inflation rate were attached. This results in four different covariate combinations 1a, 1b, 2a and 2b.

\begin{tabular}{ccccccc}
\hline Time (months) & 6 & 12 & 18 & 24 & 30 & 36 \\
\hline 1a & 0.9949 & 0.9895 & 0.9846 & 0.9807 & 0.9755 & 0.9258 \\
1b & 0.9962 & 0.9921 & 0.9884 & 0.9854 & 0.9814 & 0.9432 \\
2a & 0.9892 & 0.9780 & 0.9679 & 0.9602 & 0.9498 & 0.8618 \\
2b & 0.9919 & 0.9833 & 0.9756 & 0.9696 & 0.9616 & 0.8907 \\
\hline
\end{tabular}

Table 10: Data example. Fitted survival probabilities for multiples of six months.

\subsection{Extension: the multiple event mixture cure model}

In reality, default is not the only possible event when considering credit risk. Another event type is early repayment, which occurs when a customer repays the loan before the prede- 
fined end term. The mixture cure model could be used to repeat the exact same analysis for modeling early repayment instead of default, but it is also possible to include early repayment as an extra term in the mixture cure model (for more information on mixture cure models with multiple events, see Watkins et al., 2014; Dirick et al., 2015). For this type of models, event-specific censoring indicators $\delta_{i, d}, \delta_{i, e}$ and $\delta_{i, m}$ are introduced (denoting default, early repayment and maturity indicators respectively), along with a general censoring indicator $\delta_{i}=\delta_{i, d}+\delta_{i, e}+\delta_{i, m}$ for each observation $i$. Analogous to the susceptibility indicator $Y_{i}$, three indicators $Y_{i, d}, Y_{i, e}$ and $Y_{i, m}$ are introduced. The unconditional survival function of the multiple event mixture cure model is then given by

$S(t \mid \boldsymbol{z}, \boldsymbol{x}(t))=\pi_{e}(\boldsymbol{z}) S_{e}\left(t \mid Y_{e}=1, \boldsymbol{z}, \boldsymbol{x}(t)\right)+\pi_{d}(\boldsymbol{z}) S_{d}\left(t \mid Y_{d}=1, \boldsymbol{z}, \boldsymbol{x}(t)\right)+\left(1-\pi_{e}(\boldsymbol{z})-\pi_{d}(\boldsymbol{z})\right)$,

with $S_{e}\left(t \mid Y_{e}=1, \boldsymbol{z}, \boldsymbol{x}(t)\right)$ and $S_{d}\left(t \mid Y_{d}=1, \boldsymbol{z}, \boldsymbol{x}(t)\right)$ the conditional survival functions for early repayment and default respectively. These functions are modeled using two Cox PH models, as in (5).

Two major changes with regard to the single event mixture cure model are the computation of $\pi_{d}(\boldsymbol{z})$ and $\pi_{e}(\boldsymbol{z})$, and the conditional expectations of the $Y$-indicators, resulting in the weights $w$. With more than two groups, the binomial logit is replaced by the multinomial logit,

$$
\pi_{d}(\boldsymbol{z})=P\left(Y_{d}=1 \mid \boldsymbol{z}\right)=\frac{\exp \left(b_{d}{ }^{\prime} \boldsymbol{z}\right)}{1+\exp \left(b_{d}{ }^{\prime} \boldsymbol{z}\right)+\exp \left(b_{e}{ }^{\prime} \boldsymbol{z}\right)}
$$

and $\pi_{e}(\boldsymbol{x})$ is found analogously. As an extension to (13), the event-specific weights for early repayment and default can be computed, with in this case $\boldsymbol{\Theta}=\left(\boldsymbol{b}, \boldsymbol{\beta}_{d}, \boldsymbol{\beta}_{T, d}, \boldsymbol{\beta}_{e}, \boldsymbol{\beta}_{T, e}\right)$, and $\mathbf{O}=\left(\lambda_{d, i, j}, \lambda_{e, i, j}, \delta_{i}, \delta_{i, d}, \delta_{i, e}, \delta_{i, m}, t_{i, d}, t_{i, e}\right)$. The interval-specific censoring indicators $\lambda$ as well as the event time $t$ depend on the event type, default or early repayment. The event-specific weight for default is then given by

$$
\begin{aligned}
& w_{i, d}^{(r)}=E\left(Y_{i, d} \mid \mathbf{\Theta}^{(r)}, \mathbf{O}\right) \\
& = \begin{cases}\frac{\pi_{d}\left(\boldsymbol{z}_{i}\right) S_{d}\left(t_{i} \mid Y_{i, d}=1, \boldsymbol{z}_{i}, \boldsymbol{x}_{i}\left(t_{i}\right)\right)}{\pi_{e}\left(\boldsymbol{z}_{i}\right) S_{e}\left(t_{i} \mid Y_{i, e}=1, \boldsymbol{z}_{i}, \boldsymbol{x}_{i}\left(t_{i}\right)\right)+\pi_{d}\left(\boldsymbol{z}_{i}\right) S_{d}\left(t_{i} \mid Y_{i, d}=1, \boldsymbol{z}_{i}, \boldsymbol{x}_{i}\left(t_{i}\right)\right)+\left(1-\pi_{e}\left(\boldsymbol{z}_{i}\right)-\pi_{d}\left(\boldsymbol{z}_{i}\right)\right)} & \text { for } \delta_{i}=0 \\
1 & \text { for } \delta_{i, d}=1 . \\
0 & \text { for } \delta_{i, d}=0 ; \delta_{i}=1\end{cases}
\end{aligned}
$$

Note that, when $\delta_{i}=0, t_{i, d}=t_{i, e}=t_{i}, w_{i, e}^{(r)}$ can be computed in a similar fashion. Again, the EM-algorithm is used for computation of the expected complete-data log likelihood. 
The multiple event mixture cure model was applied to the data, adding the information about early repayments which was ignored when applying the single event mixture cure model.

Ten arbitrarily selected models containing three to four TVCs were analyzed, each time including the same TVCs for the default and early repayment events. The result for one of these models is listed in Table 11. A general result from the ten models was that, where the effect and statistical significance of $\hat{\boldsymbol{b}}$ on default lies relatively close to the results in Table 8, nearly all $\hat{\boldsymbol{\beta}}$ became statistically insignificant. The significant effects that generally remain, are the number of years at current address and the bureau score. Additionally, no significant $\hat{\boldsymbol{\beta}}_{T}$ remains for default. The signs of the early repayment parameter estimates tend to be the same as those of the default parameter. While an early repayment does not immediately incur costs for a bank, this event type does lead to a decline in expected revenue, as the interest payments for the months following the time of early repayment are lost. In fact, one can look at both default and early repayment as events that are results of a common trigger, which is customer instability. Therefore early repayment is seen as a negative event that banks prefer to avoid, and this is also reflected in the parameter estimates. For early repayment, two TVCs tend to have a significant effect on the hazard of early repayment, which is also illustrated in the model from Table 11, by the BEL20 index and the gross domestic product.

\subsection{Extension: The mixture cure model with piecewise linear relationship for the TVCs}

With abundant information on the TVCs (with one TVC-value per subject per month that the subject is observed), estimating just one $\beta_{T}$ for each TVC might be overly simplistic. On the other hand, the effect of a certain TVC on default might depend on the specific range this TVC is in. For example, the effect of the TVC associated with the GDP value might be different when the GDP is declining with respect to the previous year, compared with when GDP is increasing. A way of overcoming this is by using piecewise linear functions instead of just one linear effect (or one $\beta_{T}$ ) per TVC.

Six new models were constructed from our data, each time with the seven "baseline" 


\begin{tabular}{|c|c|c|c|c|c|c|c|c|c|c|c|c|c|}
\hline$d / e$ & (int) & $\hat{b}_{1}$ & $\hat{b}_{2}$ & $\hat{b}_{3}$ & $\hat{b}_{4}$ & $\hat{b}_{5}$ & $\hat{b}_{6}$ & $\hat{b}_{7}$ & \multicolumn{5}{|c|}{$A I C_{c d}$} \\
\hline \multirow[t]{3}{*}{$d$} & -0.033 & 0.006 & -0.014 & -0.103 & -0.037 & -0.047 & -1.246 & -1.208 & \multicolumn{5}{|c|}{98836.3} \\
\hline & $(0.163)$ & $(0.004)$ & $(0.003)$ & $(0.078)$ & $(0.004)$ & $(0.005)$ & $(0.1)$ & $(0.083)$ & & & & & \\
\hline & ns & ns & $* * *$ & ns & $* * *$ & $* * *$ & $* * *$ & $* * *$ & & & & & \\
\hline \multirow[t]{4}{*}{$e$} & 0.695 & -0.007 & -0.017 & 0.008 & -0.017 & -0.014 & -0.741 & -0.217 & & & & & \\
\hline & $(0.095)$ & $(0.002)$ & $(0.001)$ & $(0.034)$ & $(0.002)$ & $(0.002)$ & $(0.041)$ & $(0.034)$ & & & & & \\
\hline & $* * *$ & $* *$ & $* * *$ & $\mathrm{~ns}$ & $* * *$ & $* * *$ & $* * *$ & $* * *$ & & & & & \\
\hline & & $\hat{\beta}_{1}$ & $\hat{\beta}_{2}$ & $\hat{\beta}_{3}$ & $\hat{\beta}_{4}$ & $\hat{\beta}_{5}$ & $\hat{\beta}_{6}$ & $\hat{\beta}_{7}$ & IR & BEL20 & C Conf & GDP & infl rate unemp \\
\hline \multirow[t]{3}{*}{$d$} & & -0.001 & -0.001 & -0.049 & -0.007 & -0.006 & -0.198 & -0.107 & 0.039 & 0.011 & & 0.007 & 0.029 \\
\hline & & $(0.005)$ & $(0.003)$ & $(0.079)$ & $(0.004)$ & $(0.006)$ & $(0.103)$ & $(0.086)$ & $(0.038)$ & $(0.061)$ & & $(0.022)$ & $(0.042)$ \\
\hline & & ns & ns & ns & . & ns & . & ns & ns & ns & & ns & ns \\
\hline \multirow[t]{3}{*}{$e$} & & 0.002 & -0.001 & 0.037 & -0.002 & -0.001 & -0.176 & -0.136 & 0.01 & -0.056 & & 0.023 & 0.022 \\
\hline & & $(0.002)$ & $(0.001)$ & $(0.036)$ & $(0.002)$ & $(0.002)$ & $(0.042)$ & $(0.035)$ & $(0.019)$ & $(0.025)$ & & $(0.009)$ & $(0.023)$ \\
\hline & & ns & ns & ns & ns & ns & $* * *$ & $* * *$ & ns & * & & * & ns \\
\hline
\end{tabular}

Table 11: Data example. The parameter estimates of a multiple event mixture cure models containing four TVCs. $\boldsymbol{d}$ are parameter estimates related to the default event, $\boldsymbol{e}$ denotes early repayment parameter estimates.

time-independent covariates and just one of the TVCs, split into four piecewise linear functions. The TVC-part of the linear predictor in (5), $\boldsymbol{\beta}^{\prime} \boldsymbol{z}+\boldsymbol{\beta}_{T}^{\prime} \boldsymbol{x}(t)$ is replaced by several $\operatorname{TVCs} \bar{x}_{j}(t)$ for each $j=1, \ldots, 6$ :

$$
\beta_{T 1} \bar{x}_{j}(t)+\beta_{T 2}\left(\bar{x}_{j}(t)-Q_{1}\right)_{+}+\beta_{T 3}\left(\bar{x}_{j}(t)-Q_{2}\right)_{+}+\beta_{T 4}\left(\bar{x}_{j}(t)-Q_{3}\right)_{+},
$$

where $Q_{1}, Q_{2}$ and $Q_{3}$ refer to the first quantile, the second quantile (or median value) and the third quantile of all the TVC-values of the relevant macro-economic factor in the data set. The notation $(x)_{+}$denotes the value $x$ if $x>0$, or 0 otherwise. The result of this construction is that the effect of a TVC changes depending on whether the $x_{j}(t)$ are in the interval $\left[0, Q_{1}\right],\left[Q_{1}, Q_{2}\right],\left[Q_{2}, Q_{3}\right]$ or $\left[Q_{3}, Q_{4}\right]$ (respectively $\beta_{T 1}, \beta_{T 1}+\beta_{T 2}, \beta_{T 1}+\beta_{T 2}+\beta_{T 3}$ and $\left.\beta_{T 1}+\beta_{T 2}+\beta_{T 3}+\beta_{T 4}\right)$.

In Table 12, this principle is illustrated using the interest rate. While this model shows that the effect between interest rate and default hazard rate is negative in the interval $\left[0, Q_{1}\right]$, the effect is positive (as would be expected) in all other intervals (the effects are $-0.064,0.404,0.343$ and 0.034 respectively). The effect of the middle ranges of the interest rate seems to be more distinct $(0.404,0.343)$ compared to the "border intervals". However, it should be noted that from the results, no conclusions can be drawn, as none of the estimates are statistically significant. Other TVCs were examined using this method as 


\begin{tabular}{c|cccccccc|cccc|c}
\hline & $($ int $)$ & $z_{1}$ & $z_{2}$ & $z_{3}$ & $z_{4}$ & $z_{5}$ & $z_{6}$ & $z_{7}$ & $\hat{\beta}_{T 1}$ & $\hat{\beta}_{T 2}$ & $\hat{\beta}_{T 3}$ & $\hat{\beta}_{T 4}$ & $A I C_{c d}$ \\
\hline $\boldsymbol{b}$ & 4.019 & 0.05 & 0.041 & 0.062 & 0.005 & -0.066 & -1.506 & -1.95 & & & & & 27484.3 \\
& $(0.084)$ & $(0.002)$ & $(0.002)$ & $(0.035)$ & $(0.002)$ & $(0.002)$ & $(0.039)$ & $(0.034)$ & & & & \\
\\
\multirow{3}{*}{$\boldsymbol{\beta}$} & $* * *$ & $* * *$ & $* * *$ &. & $* *$ & $* * *$ & $* * *$ & $* * *$ & & & & \\
& -0.007 & -0.02 & -0.131 & -0.033 & -0.02 & -0.574 & -0.351 & -0.064 & 0.468 & -0.061 & -0.309 & \\
& $(0.004)$ & $(0.003)$ & $(0.079)$ & $(0.003)$ & $(0.006)$ & $(0.101)$ & $(0.083)$ & $(0.057)$ & $(0.344)$ & $(0.509)$ & $(0.39)$ & \\
& & $* * *$ &. & $* * *$ & $* * *$ & $* * *$ & $* * *$ & $\mathrm{~ns}$ & $\mathrm{~ns}$ & $\mathrm{~ns}$ & $\mathrm{~ns}$ & \\
\hline
\end{tabular}

Table 12: Data example. The parameter estimates of mixture cure models containing the TVC interest rate, split up into four piecewise linear pieces bounded by the quantiles of the interest rate, as expressed in (11).

well, but they all lacked statistical significance hence these results are not included in this paper.

\section{Discussion}

The main reason and motivation for using the mixture cure models is that the common assumption of survival analysis, that the survival function goes to zero as time goes to infinity, clearly does not hold in many practical application settings (e.g. credit risk modeling, fraud prediction, churn detection) as illustrated in Figure 1. We have shown that time-dependent covariates can be included in mixture cure models to address this problem whilst also enabling our models to include macroeconomic conditions.

A general result we found for the data set we used is that only a limited number of macro-economic factors tended to have an effect on default (in the single event mixture cure model) and early repayment (in the multiple event mixture cure model). Where the BEL20 index had an influence on both event types, the interest rate had an influence on the former and GDP on the latter event type only. It is indeed plausible that some macroeconomic factors do not affect default or early repayment. Let us take the unemployment rate as an example: because of a selection bias (as banks only granted loans to supposedly creditworthy customers), the debtors in the data set might not be affected by higher unemployment, if a rise in unemployment was not present among the subjects in the data set. On the other hand, some actual effects of TVC on default might be lost as a result 
of the averaging of TVCs over a monthly period. Interesting results might be obtained by applying the models when looking at weekly or even daily TVC levels; however, this would largely increase the data size, and requires daily information regarding default and early repayment events.

De Leonardis and Rocci (2014) approached the mixture cure models from a discrete time perspective and applied this to study the default of firms. In that paper they work only with the observed data likelihood. This simplification allows them to avoid the use of the EM algorithm. An extension of the discrete time model of De Leonardis and Rocci (2014) to incorporate multiple events, i.e. default, early repayment and maturity, would be interesting for further research.

Several extensions of the basic model are possible. Piecewise linear functions can model more complex relationships between the TVCs and the event of interest. An interesting future research focus is setting appropriate "knots" instead of the quantile values.

As the goal of this study was to include time-dependent covariates in the latency part of the already existing (and popular) mixture cure model, we did not explore time-dependent incidence models. In fact, this is not straightforward as we are using a logistic regression model. A related yet different type of model that accommodates for time-dependency in both model parts is the promotion time cure model (Yakovlev et al., 1993). Its use for default modeling is an interesting topic for further research, as are the use of an accelerated failure time model as well as a Bayesian modeling approach.

Our extension of mixture cure models with time varying covariates is relevant in other settings too. In a fraud analytics setting, where not all customers become fraudsters, our approach will allow better disentanglement of the time varying tactics adopted by fraudsters which may lead to better fraud prevention mechanisms. In a churn prediction setting, where some customers may end up never churning, the inclusion of TVCs can enable an understanding of latent and time varying symptoms of customer dissatisfaction.

To summarize, we think there are many application settings with high censoring rates caused by the fact that the event does not occur for a majority of subjects. We believe our extension of mixture cure models with time varying covariates is a valuable tool to better understand the time varying nature of the events studied. 


\section{Acknowledgements}

We wish to thank all reviewers of this paper for their helpful suggestions and comments. We acknowledge the support of the Fund for Scientific Research Flanders, KU Leuven grant GOA/12/14 and of the IAP Research Network P7/06 of the Belgian Science Policy. The computational resources and services used in this work were provided by the VSC (Flemish Supercomputer Center), funded by the Hercules Foundation and the Flemish Government - department EWI.

\section{A Appendix. Implementation using the EM-algorithm}

\section{A.1 The E-step}

Denote the parameter-triplet $\left(\boldsymbol{b}, \boldsymbol{\beta}, \boldsymbol{\beta}_{T}\right)$ by $\Theta$, and the observed information for each observation $\left(\lambda_{i, j}, \delta_{i}, t_{i}\right)$ by $\mathbf{O}$. The conditional expectation of the complete-data log likelihood (formula (6)) in the $(r+1)$ th E-step is given by

$$
Q\left(\Theta^{(r+1)} \mid \Theta^{(r)}\right)=E\left[\log L_{c}\left(\Theta^{(r+1)} \mid \boldsymbol{z}, \boldsymbol{x}(t), Y\right) \mid \Theta^{(r)}, \mathbf{O}\right] .
$$

It can easily be seen that these functions are linear in $Y_{i}$, which reduces the problem to find an expression for the conditional expectation of $Y_{i}$, which is given by

$$
w_{i}^{(r)}=E\left(Y_{i} \mid \mathbf{\Theta}^{(r)}, \mathbf{O}\right)= \begin{cases}\frac{\pi\left(\boldsymbol{z}_{i}\right) S\left(t_{i} \mid Y_{i}=1, \boldsymbol{z}_{i}, \boldsymbol{x}_{i}\left(t_{i}\right)\right)}{\pi\left(\boldsymbol{z}_{i}\right) S\left(t_{i} \mid Y_{i}=1, \boldsymbol{z}_{i}, \boldsymbol{x}_{i}\left(t_{i}\right)\right)+\left(1-\pi\left(\boldsymbol{z}_{i}\right)\right)} & \text { for } \delta_{i}=0 \\ 1 & \text { for } \delta_{i}=1 .\end{cases}
$$

Note that $E\left(Y_{i} \mid \mathbf{\Theta}^{(r)}, \mathbf{O}\right)$ takes one value per iteration for each observation. The weights $w_{i}^{(r)}$ are computed using the value of the TVCs at time of censoring, and can be interpreted as the probability that individual $i$ will be susceptible to the event.

\section{A.2 The M-step}

The expected complete-data log likelihood in (12) is maximized with respect to the unknown parameters. The conditional expectation of the incidence log likelihood is straightforward, replacing $Y_{i}^{\prime}$ 's in (7) by $w_{i}^{(r)}$. The conditional expectation of the latency $\log$ 
likelihood (8) equals, using $\lambda_{i, j} \log w_{i}^{(r)}=0$ and $\lambda_{i, j} w_{i}^{(r)}=\lambda_{i, j}$ (Cai et al., 2012a)

$$
\begin{aligned}
E & {\left[\log L_{\text {lat }}\left(\boldsymbol{\beta}, \boldsymbol{\beta}_{T} \mid \boldsymbol{z}, \boldsymbol{x}(t), Y\right) \mid \boldsymbol{\Theta}^{(r)}, \mathbf{O}\right] } \\
& =\sum_{i=1}^{n} \sum_{j=1}^{k_{i}} \lambda_{i, j} \log \left(w_{i}^{(r)} h\left(t_{j} \mid Y_{i}=1, \boldsymbol{z}_{i}, \boldsymbol{x}_{i}\left(t_{j}\right)\right)\right)+w_{i}^{(r)} \log S\left(t_{j} \mid Y_{i}=1, \boldsymbol{z}_{i}, \boldsymbol{x}_{i}\left(t_{j}\right)\right) \\
& =\log \prod_{i=1}^{n} \prod_{j=1}^{k_{i}}\left(w_{i}^{(r)} h_{0}\left(t_{j}\right) \exp \left(\boldsymbol{\beta}^{\prime} \boldsymbol{z}_{i}+\boldsymbol{\beta}_{T}^{\prime} \boldsymbol{x}_{i}\left(t_{j}\right)\right)\right)^{\lambda_{i, j}}\left(S_{0}\left(t_{j}\right)^{\exp \left(\boldsymbol{\beta}^{\prime} \boldsymbol{z}_{i}+\boldsymbol{\beta}_{T}^{\prime} \boldsymbol{x}_{i}\left(t_{j}\right)\right)}\right)^{w_{i}^{(r)}} \\
& =\log \prod_{i=1}^{n} \prod_{j=1}^{k_{i}}\left(h_{0}\left(t_{j}\right) \exp \left(\boldsymbol{\beta}^{\prime} \boldsymbol{z}_{i}+\boldsymbol{\beta}_{T}^{\prime} \boldsymbol{x}_{i}\left(t_{j}\right)+\log w_{i}^{(r)}\right)\right)^{\lambda_{i, j}}\left(S_{0}\left(t_{j}\right)^{\exp \left(\boldsymbol{\beta}^{\prime} \boldsymbol{z}_{i}+\boldsymbol{\beta}_{T}^{\prime} \boldsymbol{x}_{i}\left(t_{j}\right)+\log w_{i}^{(r)}\right)}\right) .
\end{aligned}
$$

When (12) is maximized, the baseline survival function of the $r^{\text {th }}$ M-step is updated before proceeding with the next E-step. This is done non-parametrically using the Breslow-type estimator for $S_{0}(t)$ and combining the results of Andersen (1992) and Cai et al. (2012a). Denote $R\left(t_{j}\right)$ the individuals at risk in the interval $\left(B_{j-1}, B_{j}\right]$, then

$$
\hat{S}_{0}(t)=\exp \left(-\sum_{j: t_{j} \leq t} \frac{\sum_{i \in R\left(t_{j}\right)}^{n} \lambda_{i, j}}{\sum_{i \in R\left(t_{j}\right)}^{n} w_{i}^{(r)} \exp \left(\boldsymbol{\beta}^{\prime(r)} \boldsymbol{z}_{i}+\boldsymbol{\beta}_{T}^{(r)} \boldsymbol{x}_{i}\left(t_{j}\right)\right)}\right) .
$$

The E-step and the M-step are repeated until parameter convergence.

\section{A.3 Variance estimation}

Standard errors of parameter estimators obtained via an EM-algorithm are not directly available. A widespread method for estimating variances in the mixture cure context is bootstrapping (e.g. Peng, 2003; Cai et al., 2012a; Tong et al., 2012). While easy to implement, this method is computationally expensive, especially with big data sets and a slow convergence of the EM-algorithm. We use the supplemented EM (SEM) algorithm introduced by Meng and Rubin (1991). While other approximation methods exist (see, among others, Sy and Taylor (2000); Peng and Dear (2000)) the advantage of SEM is that it can be applied to any problem to which EM is applied, assuming that there is access to the complete-data asymptotic variance-covariance matrix, which is indeed the case here. 


\section{References}

Andersen, P. K. (1992). Repeated assessment of risk factors in survival analysis. Statistical Methods in Medical Research, 1(3):297-315.

Austin, P. C. (2012). Generating survival times to simulate cox proportional hazards models with time-varying covariates. Statistics in medicine, 31(29):3946-3958.

Banasik, J., Crook, J., and Thomas, L. (1999). Not if but when will borrowers default. The Journal of the Operational Research Society, 50(12):1185-1190.

Bellotti, T. and Crook, J. (2009). Credit scoring with macroeconomic variables using survival analysis. The Journal of the Operational Research Society, 60(12):1699-1707.

Berkson, J. and Gage, R. P. (1952). Survival curve for cancer patients following treatment. Journal of the American Statistical Association, 47(259):501-515.

Cai, C., Zou, Y., Peng, Y., and Zhang, J. (2012a). smcure: An R-package for estimating semiparametric mixture cure models. Computer Methods and Programs in Biomedicine, 108:1255-1260.

Cai, C., Zou, Y., Peng, Y., and Zhang, J. (2012b). smcure: Fit Semiparametric Mixture Cure Models. R package version 2.0.

Claeskens, G. and Hjort, N. L. (2008). Model Selection and Model Averaging. Cambridge University Press.

Collett, D. (2003). Modelling Survival Data in Medical Research, Second Edition. Chapman \& Hall/CRC Texts in Statistical Science. CRC Press.

Cortese, G. and Andersen, P. K. (2010). Competing risks and time-dependent covariates. Biometrical Journal, 52(1):138-158.

Cox, D. and Oakes, D. (1984). Analysis of Survival Data. Chapman \& Hall/CRC Monographs on Statistics \& Applied Probability. Taylor \& Francis.

Crook, J. and Bellotti, T. (2010). Time varying and dynamic models for default risk in consumer loans. Journal of the Royal Statistical Society: Series A (Statistics in Society), 173(2):283-305.

Danilov, D. and Magnus, J. R. (2004). On the harm that ignoring pretesting can cause. Journal of Econometrics, 122(1):27-46.

De Leonardis, D. and Rocci, R. (2014). Default risk analysis via a discrete-time cure rate 
model. Applied Stochastic Models in Business and Industry, 30(5):529-543.

Dempster, A. P., Laird, N. M., and Rubin, D. B. (1977). Maximum likelihood from incomplete data via the em algorithm. Journal of the Royal Statistical Society. Series B (Methodological), pages 1-38.

Dirick, L., Claeskens, G., and Baesens, B. (2015). An Akaike information criterion for multiple event mixture cure models. European Journal of Operational Research, 241:449457.

Divino, J. A. and Rocha, L. C. S. (2013). Probability of default in collateralized credit operations. The North American Journal of Economics and Finance, 25:276 - 292.

Farewell, V. T. (1982). The use of mixture models for the analysis of survival data with long-term survivors. Biometrics, 38(4):1041-1046.

Fisher, L. D. and Lin, D. Y. (1999). Time-dependent covariates in the Cox proportionalhazards regression model. Annual review of public health, 20(1):145-157.

Fox, J. (2002). Cox proportional-hazards regression for survival data. An $R$ and $S$-PLUS companion to applied regression, pages 1-18.

Fox, J. and Carvalho, M. S. (2012). The RcmdrPlugin.survival package: Extending the R commander interface to survival analysis. Journal of Statistical Software, 49(7):1-32.

Hosmer, D. W., May, S., and Lemeshow, S. (2008). Applied Survival Analysis: Regression Modelling of Time to Event Data. Wiley-Interscience, second edition.

Kalbfleisch, J. and Prentice, R. (2002). The Statistical Analysis of Failure Time Data. John Wiley \& Sons, New Jersey, second edition.

Kuk, A. and Chen, C. (1992). A mixture model combining logistic regression with proportional hazards regression. Biometrika, 79(3):531-541.

Meng, X.-L. and Rubin, D. B. (1991). Using EM to obtain asymptotic variancecovariance matrices: The SEM algorithm. Journal of the American Statistical Association, 86(416):899-909.

Narain, B. (1992). Survival analysis and the credit granting decision. In Thomas, L. C., Crook, J. N., and Edelman, D. B., editors, Credit Scoring and Credit Control, pages 109-121. Clarendon Press, Oxford.

NBB (2015). National Bank of Belgium: Database with macro-economic factors. http: 
//stat.nbb.be.

Peng, Y. (2003). Fitting semiparametric cure models. Computational Statistics \& Data Analysis, 41(3-4):481 - 490. Recent Developments in Mixture Model.

Peng, Y. and Dear, K. (2000). A nonparametric mixture model for cure rate estimation. Biometrics, 56(1):227-236.

R Core Team (2013). R: A Language and Environment for Statistical Computing. $\mathrm{R}$ Foundation for Statistical Computing, Vienna, Austria. ISBN 3-900051-07-0.

Stepanova, M. and Thomas, L. (2002). Survival analysis methods for personal loan data. Operations Research Quarterly, 50(2):277-289.

Sy, J. and Taylor, J. (2000). Estimation in a Cox proportional hazards cure model. Biometrics, 56(1):227-236.

Therneau, T. M. (2014). A Package for Survival Analysis in S. R package version 2.37-7.

Tong, E. N. C., Mues, C., and Thomas, L. C. (2012). Mixture cure models in credit scoring: if and when borrowers default. European Journal of Operational Research, 218(1):132139.

Van Gestel, T. and Baesens, B. (2008). Credit Risk Management : Basic Concepts: Financial Risk Components, Rating Analysis, Models, Economic and Regulatory Capital. OUP Oxford.

Watkins, J. G. T., Vasnev, A. L., and Gerlach, R. (2014). Multiple event incidence and duration analysis for credit data incorporating non-stochastic loan maturity. Journal of Applied Econometrics, 29:627-648.

Yakovlev, A. Y., Asselain, B., Bardou, V., Fourquet, A., Hoang, T., Rochefediere, A., and Tsodikov, A. (1993). A simple stochastic model of tumor recurrence and its application to data on premenopausal breast cancer. Biometrie et analyse de donnees spatio-temporelles, 12:66-82. 